\title{
Determining the First Probability Density Function of Linear Random Initial Value Problems by the Random Variable Transformation (RVT) Technique: A Comprehensive Study
}

\author{
M.-C. Casabán, J.-C. Cortés, J.-V. Romero, and M.-D. Roselló \\ Instituto Universitario de Matemática Multidisciplinar, Universitat Politècnica de València, Camino de Vera s/n, 46022 \\ Valencia, Spain \\ Correspondence should be addressed to J.-V. Romero; jvromero@imm.upv.es
}

Received 20 June 2013; Accepted 14 October 2013; Published 21 January 2014

Academic Editor: G. M. N’Guérékata

Copyright (C) 2014 M.-C. Casabán et al. This is an open access article distributed under the Creative Commons Attribution License, which permits unrestricted use, distribution, and reproduction in any medium, provided the original work is properly cited.

\begin{abstract}
Deterministic differential equations are useful tools for mathematical modelling. The consideration of uncertainty into their formulation leads to random differential equations. Solving a random differential equation means computing not only its solution stochastic process but also its main statistical functions such as the expectation and standard deviation. The determination of its first probability density function provides a more complete probabilistic description of the solution stochastic process in each time instant. In this paper, one presents a comprehensive study to determinate the first probability density function to the solution of linear random initial value problems taking advantage of the so-called random variable transformation method. For the sake of clarity, the study has been split into thirteen cases depending on the way that randomness enters into the linear model. In most cases, the analysis includes the specification of the domain of the first probability density function of the solution stochastic process whose determination is a delicate issue. A strong point of the study is the presentation of a wide range of examples, at least one of each of the thirteen casuistries, where both standard and nonstandard probabilistic distributions are considered.
\end{abstract}

\section{Introduction and Motivation}

Over the last few decades, random differential equations (RDEs) have been demonstrated to be powerful tools to model numerous problems appearing in many different areas such as physics, engineering, economics, epidemiology, and hydrology. The consideration of randomness into their formulation through initial/boundary conditions, source terms, and/or coefficients adapts better than their deterministic counterpart to model the uncertainty associate to the experimental measurement required to set the above inputs as well as the inherent complexity involved in many real modelling problems. This approach leads to face new and exciting questions different from the corresponding ones appearing in the deterministic scenario. Indeed, instead of obtaining just the solution stochastic process (SP) of RDEs, the theory is also concerned with its probabilistic properties, mainly the computations of the expectation and variance functions. The computation of the first probability density function (1-PDF) of the solution SP, say $x(t)$, is much more desirable since, from it, one can compute the previous statistical functions as simple particular cases and, in addition, it provides a comprehensive probabilistic description of the solution SP for each time instant $t$. However, the computation of the 1-PDF constitutes a major challenge that can been achieved in only a few cases.

The aim of this paper is to determine the 1-PDF, $f_{1}(z, t)$, of the solution SP $Z(t)$ to the linear random initial value problem (IVP):

$$
\begin{gathered}
\dot{Z}(t)=A Z(t)+B, \quad t>t_{0}, \\
Z\left(t_{0}\right)=Z_{0},
\end{gathered}
$$

where the data $Z_{0}, B$ and $A$ are assumed to be continuous random variables (continuous RVs) defined on a common 
probability space $(\Omega, \mathscr{F}, \mathbb{P})$, whose domains are assumed to be

$$
\begin{gathered}
D_{Z_{0}}=\left\{z_{0}=Z_{0}(\omega), \omega \in \Omega: z_{0,1} \leq z_{0} \leq z_{0,2}\right\}, \\
D_{B}=\left\{b=B(\omega), \omega \in \Omega: b_{1} \leq b \leq b_{2}\right\}, \\
D_{A}=\left\{a=A(\omega), \omega \in \Omega: a_{1} \leq a \leq a_{2}\right\},
\end{gathered}
$$

respectively. Hereinafter, in order to avoid cumbersome notation, we will hide the $\omega$ sample dependence when writing domains of continuous RVs. In this way, for instance, the domain $D_{Z_{0}}$ will be written as $D_{Z_{0}}=\left\{z_{0}: z_{0,1} \leq z_{0} \leq z_{0,2}\right\}$ rather than the first expression in (2). The same can be said for $D_{B}, D_{A}$, and the domain of any other RV throughout this paper. We allow the left (right) endpoint of each interval of the domain takes the value $-\infty(+\infty)$; that is, we also consider unbounded continuous RVs. Throughout the paper, we will denote by $f_{Z_{0}}\left(z_{0}\right), f_{B}(b)$, and $f_{A}(a)$ the PDFs of the continuous RVs $Z_{0}, B$, and $A$, respectively. The case where $Z_{0}$, $B$ and $A$ are pairwise dependent continuous RVs will also be treated. In such case, $f_{Z_{0}, B}\left(z_{0}, b\right), f_{Z_{0}, A}\left(z_{0}, a\right)$, and $f_{B, A}(b, a)$ will denote the joint PDFs of the random vectors: $\left(Z_{0}, B\right)$, $\left(Z_{0}, A\right)$, and $(B, A)$, respectively. Finally, we will also deal with the case where $Z_{0}, B$, and $A$ are dependent continuous RVs, then $f_{Z_{0}, B, A}\left(z_{0}, b, a\right)$ will represent their joint PDF. Notice that the domains of these two- and three-dimensional PDFs often can be written directly as products of the sets $D_{Z_{0}}, D_{B}$, and $D_{A}$ given by (2).

In order to compute the 1-PDF $f_{1}(z, t)$, random variable transformation (RVT) method will be applied. RVT is a probability technique that allows us to calculate the PDF $f_{Y}(y)$ of a RV $Y$ resulting after the algebraic transformation of another RV, say $X$, whose PDF, $f_{X}(x)$, is known. In its simplest scalar formulation, the method reads as follows: if $X$ is a continuous RV lying on the domain or support $D_{X}=$ $\left\{x: x_{1} \leq x \leq x_{2}\right\}$, whose PDF is $f_{X}(x)>0$ and $Y=r(X)$ being $r: D_{X} \subseteq \mathbb{R} \rightarrow \mathbb{R}$ a monotone mapping on $D_{X}$, then

$$
f_{Y}(y)=f_{X}(s(y))\left|\frac{\mathrm{d} s(y)}{\mathrm{d} y}\right|, \quad D_{Y}=\left\{y: y_{1} \leq y \leq y_{2}\right\},
$$

where $s(y)=x$ is the inverse function of $r$ on $D_{X}$, which is assumed to have a continuous derivative on $D_{Y}$ and $|\mathrm{d} s(y) / \mathrm{d} y|$ denotes the modulus of the derivative of $s(y)$. In the particular case that $r$ increases (decreases) on $D_{X}$, the domain $D_{Y}$ of $Y=r(X)$ is determined by $D_{Y}=\left\{y: y_{1}=\right.$ $\left.r\left(x_{1}\right) \leq y \leq r\left(x_{2}\right)=y_{2}\right\}\left(D_{Y}=\left\{y: y_{1}=r\left(x_{2}\right) \leq y \leq r\left(x_{1}\right)=\right.\right.$ $\left.y_{2}\right\}$.

Notice that we are interested in computing the 1-PDF $f_{1}(z, t)$ to the solution $Z(t)$ of (1) which is a SP rather than a RV, whereas RVT technique is mainly designed to handle (transformations of) continuous RVs. In order to take advantage of RVT, we first will fix $\widehat{t}: \hat{t}>t_{0}$ and then we will apply RVT to the (transformed) RV $Z(\hat{t})$. Therefore, we can say that RVT technique provides a time-transversal description of the 1-PDF $f_{1}(z, t)$.

Some of the earliest applications of RVT method to RDEs can be found in $[1, \mathrm{ch} .6]$ where this technique is applied to study a linear oscillator assuming randomness just in the two initial conditions related to the position and velocity. Most of the subsequent contributions have focused on the study of particular equations assuming specific probabilistic distributions for the involved uncertainty which facilitates the analysis. Here, we point out some recent contributions that illustrate quite well the current trends of RVT method in dealing with RDEs. In [2], authors solve the radiative transfer equation in a semi-infinite continuous stochastic medium with Rayleigh scattering. RVT method is applied to obtain the 1-PDF of the solution when the optical depth space variable is assumed to be a RV belonging to the following particular distributions: exponential and Gaussian. Higher order statistical moments of the solution stochastic process are also computed. An analogous study on the stochastic transport equation of neutral particles with anisotropic scattering can be found in [3]. In $[4,5]$, authors apply RVT technique to develop a stochastic finite element method for solving some stochastic problems with random excitation.

The application of RVT technique to the exact determination of the 1-PDF of the solution SP of RDEs requires the previous computation of the exact solution of the RDE under study. However, in the outstanding contribution [6], author takes advantage of RVT method together with classical numerical techniques to illustrate, through a wide range of examples, the potentiality of this method to approximate the 1-PDF for the solution SP of some RDEs.

As it has been announced previously, in this paper, we will compute the 1-PDF of the IVP (1) whose exact solution is available. For the sake of clarity, in the presentation, we will divide the study in the three main IVPs (I)-(III) listed in Table 1. In Case (I), we consider the homogeneous $(\mathrm{H})$ problem whereas Cases (II) and (III) deal with the nonhomogeneous (NH) cases. Within each case, we distinguish, in a systematic manner, the different possibilities regarding the randomness of each of the involved parameters $Z_{0}, B$, and $A$. These casuistries include the situations where the parameters are statistically dependent. In this context, and as it has been pointed out previously, if for example $Z_{0}$ and $B$ are statistically dependent, then $f_{Z_{0}, B}\left(z_{0}, b\right)$ will denote the joint PDF of the random vector $\left(Z_{0}, B\right)$, and the same can be said for the rest of the possibilities. The IVPs (I) and (II) can be seen as particular cases of the IVP (III) when $\mathbb{P}[\{\omega \in \Omega: B(\omega)=0\}]=1$ and $\mathbb{P}[\{\omega \in \Omega: A(\omega)=0\}]=1$, respectively. When uncertainty can only be attributted to $Z_{0}$ and $B$, and the parameter $A$ can be set in a deterministic way, it is more realistic and convenient to assume that the joint $\operatorname{PDF} f_{Z_{0}, B}\left(z_{0}, b\right)$ is known rather than $f_{Z_{0}, B, A}\left(z_{0}, b, a\right)$. Notice that the construction of the joint PDF of only two continuous RVs from measured data can become a difficult problem which accuracy can deteriorate severely if one includes a new and inappropriate RV into its formulation [7]. The most accuracy of the PDF of the random input parameter, the best approximation of the 1-PDF of the solution SP of the IVP (1). Therefore, the consideration of all the thirteen separate cases listed in Table 1 turns out more recommendable from a practical point of view.

For the sake of clarity in the development of all the cases listed in Table 1, throughout this paper, the input parameters, 
TABLE 1: List of the thirteen different cases considered to perform the full study depending on whether the IVP is homogeneous (H) or nonhomogeneous $(\mathrm{NH})$ and the form that uncertainty is considered into the problem: just through one RV: $Z_{0}, B$, or $A$ (Cases I-II-III.1, I-II-III.2, and III.3), two RVs: $\left(Z_{0}, B\right),\left(Z_{0}, A\right)$, or $(B, A)$ (Cases I-II.3 and III.4-5-6), and the three involved RVs: $\left(Z_{0}, B, A\right)($ Case III.7).

\begin{tabular}{|c|c|c|c|c|}
\hline Type & IVP & & & \\
\hline \multirow{3}{*}{$\mathrm{H}$} & \multirow{3}{*}{$\begin{array}{c}\dot{Z}(t)=A Z(t) \\
Z\left(t_{0}\right)=Z_{0}\end{array}$} & \multirow{3}{*}{$(\mathrm{I})$} & Case I.1 & $Z_{0}$ is a random variable \\
\hline & & & Case I.2 & $A$ is a random variable \\
\hline & & & Case I.3 & $\left(Z_{0}, A\right)$ is a random vector \\
\hline \multirow{10}{*}{$\mathrm{NH}$} & \multirow{3}{*}{$\begin{aligned} \dot{Z}(t) & =B \\
Z\left(t_{0}\right) & =Z_{0}\end{aligned}$} & \multirow{3}{*}{ (II) } & Case II.1 & $Z_{0}$ is a random variable \\
\hline & & & Case II.2 & $B$ is a random variable \\
\hline & & & Case II.3 & $\left(Z_{0}, B\right)$ is a random vector \\
\hline & \multirow{7}{*}{$\begin{array}{c}\dot{Z}(t)=A Z(t)+B \\
Z\left(t_{0}\right)=Z_{0}\end{array}$} & \multirow{7}{*}{ (III) } & Case III.1 & $Z_{0}$ is a random variable \\
\hline & & & Case III.2 & $B$ is a random variable \\
\hline & & & Case III.3 & $A$ is a random variable \\
\hline & & & Case III.4 & $\left(Z_{0}, B\right)$ is a random vector \\
\hline & & & Case III.5 & $\left(Z_{0}, A\right)$ is a random vector \\
\hline & & & Case III.6 & $(B, A)$ is a random vector \\
\hline & & & Case III.7 & $\left(Z_{0}, B, A\right)$ is a random vector \\
\hline
\end{tabular}

which are assumed to be continuous RVs, will be denoted by upper cases, while deterministic magnitudes will be written by lower cases. More precisely, for instance, in Case III.4, we will denote by $Z_{0}$ and $B$ the random inputs, while the multiplicative coefficient in the RDE will be denoted by $a$ rather than $A$.

The paper is organized as follows. In Section 2 we summarize the main results concerned with RVT that will be applied throughout the paper. We particularly state different versions of this useful technique including its application to different transformations that will facilitate the presentation of the results. Sections 3, 4, and 5 provide a detailed study where the 1-PDF of the solution SP, $Z(t)$, corresponding to the IVPs (I), (II), and (III) listed in Table 1, respectively, is computed. For each one of the thirteen casuistries, the study shows, at least, an illustrative example. The choice of the PDFs considered in the examples has been made to show the ability of the method to deal with both standard and non-standard dependent probability distributions. In Section 6 we include some considerations related to the application and better understanding of RVT method that we found particularly useful. Conclusions are drawn in the closing section.

\section{Preliminaries}

Below, we state several versions of the RVT technique as well as some related results emerging from its applications that will play a relevant role in our subsequent developments. Most of these results can be found in $[8,9]$ or they are a direct consequence of them.

Theorem 1 (RVT technique: scalar version). Let $X$ be $a$ continuous $R V$ with PDF $f_{X}(x)$ and domain $D_{X}=\{x$ : $\left.f_{X}(x)>0\right\}$. Let $Y=r(X)$ be a new $R V$ generated by the map $r$ : $\mathbb{R} \rightarrow \mathbb{R}$ which is assumed to be continuously differentiable on $D_{X}$ and such that $r^{\prime}(x) \neq 0$ except at a finite number of points.
Let one suppose that, for each $y \in \mathbb{R}$, there exist $m(y) \geq 1$ points: $x_{1}(y), x_{2}(y), \ldots, x_{m(y)}(y) \in D_{X}$ such that

$$
r\left(x_{k}(y)\right)=y, \quad r^{\prime}\left(x_{k}(y)\right) \neq 0, \quad k=1,2, \ldots, m(y) .
$$

Then

$$
f_{Y}(y)= \begin{cases}\sum_{i=1}^{m(y)} f_{X}\left(x_{k}(y)\right)\left|r^{\prime}\left(x_{k}(y)\right)\right|^{-1} & \text { if } m(y)>0, \\ 0 & \text { if } m(y)=0 .\end{cases}
$$

Although Theorem 1 unifies the treatment of the different cases that one can present to compute the PDF, $f_{Y}(y)$, in practice, it is easier to determine $f_{Y}(y)$ by dividing the domain of RV $X$ into subintervals where the mapping $r$ is monotone and then applying formula (3) on each subinterval. The process to compute $f_{Y}(y)$ on the whole domain of $Y$ is completed by adding the corresponding expressions calculated previously for each subinterval.

In the simplest but significant case where the map $r$ is linear, Theorem 1 reads as follows.

Proposition 2 (RVT technique: linear transformation). Let $X$ be a continuous $R V$ with domain $D_{X}=\left\{x: x_{1} \leq\right.$ $\left.x \leq x_{2}\right\}$ and PDF $f_{X}(x)$. Then, the PDF $f_{Y}(y)$ of the linear transformation $Y=\alpha X+\beta, \alpha \neq 0$ is given by

$$
\begin{aligned}
& f_{Y}(y)=\frac{1}{|\alpha|} f_{X}\left(\frac{y-\beta}{\alpha}\right), \\
& \text { where } \begin{cases}y_{1}=\alpha x_{1}+\beta \leq y \leq \alpha x_{2}+\beta=y_{2} & \text { if } \alpha>0, \\
y_{1}=\alpha x_{2}+\beta \leq y \leq \alpha x_{1}+\beta=y_{2} & \text { if } \alpha<0 .\end{cases}
\end{aligned}
$$

If $\alpha=0$, then $Y=\beta$ w.p. 1 stands for with probability 1 and

$$
f_{Y}(y)=\delta(y-\beta), \quad-\infty<y<\infty,
$$

where $\delta(\cdot)$ denotes the Dirac delta distribution. 
The following result is a direct application of Theorem 1 in the case that $r(x)=\alpha \exp (\beta x)+\gamma$.

Proposition 3 (RVT technique: exponential transformation). Let $X$ be a continuous $R V$ with domain $D_{X}=\left\{x: x_{1} \leq x \leq\right.$ $\left.x_{2}\right\}$ and $\operatorname{PDF} f_{X}(x)$. Then the PDF $f_{Y}(y)$ of the exponential transformation $Y=\alpha \exp (\beta X)+\gamma$, with $\alpha \beta \neq 0$, is given by

$$
\begin{aligned}
& f_{Y}(y)=\frac{1}{|\beta(y-\gamma)|} f_{X}\left(\frac{1}{\beta} \ln \left(\frac{y-\gamma}{\alpha}\right)\right), \\
& \text { where }\left\{\begin{array}{l}
y_{1}=\alpha \mathrm{e}^{\beta x_{1}}+\gamma \leq y \leq \alpha \mathrm{e}^{\beta x_{2}}+\gamma=y_{2} \\
y_{1}=\alpha \mathrm{e}^{\beta x_{2}}+\gamma \leq y \leq \alpha \mathrm{e}^{\beta x_{1}}+\gamma=y_{2}
\end{array} \text { if } \alpha \beta>0,\right.
\end{aligned}
$$

$$
\text { If } \alpha=0 \text { or } \beta=0 \text {, then } Y=\alpha+\gamma \text { with probability } 1 \text { and }
$$

$$
f_{Y}(y)=\delta(y-(\alpha+\gamma)), \quad-\infty<y<\infty .
$$

The computation of the joint PDF of two or more continuous RVs using the RVT method can also be performed by using the following generalization of formula (3).

Theorem 4 (RVT technique: multidimensional version). Let $\mathbf{X}=\left(X_{1}, \ldots, X_{n}\right)$ be a random vector of dimension $n$ with joint PDF $f_{\mathbf{X}}(\mathbf{x})$. Let $\mathbf{r}: \mathbb{R}^{n} \rightarrow \mathbb{R}^{n}$ be a one-to-one deterministic map which is assumed to be continuous with respect to each one of its arguments and with continuous partial derivatives. Then, the joint PDF $f_{\mathbf{Y}}(\mathbf{y})$ of the random vector $\mathbf{Y}=\mathbf{r}(\mathbf{X})$ is given by

$$
f_{\mathbf{Y}}(\mathbf{y})=f_{\mathbf{X}}(\mathbf{s}(\mathbf{y}))\left|J_{n}\right|
$$

where $\mathbf{s}(\mathbf{y})$ is the inverse transformation of $\mathbf{r}(\mathbf{x}): \mathbf{x}=\mathbf{r}^{-1}(\mathbf{y})=$ $\mathbf{s}(\mathbf{y})$ and $J_{n}$ is the Jacobian of the transformation; that is,

$$
J_{n}=\operatorname{det}\left(\frac{\partial \mathbf{x}}{\partial \mathbf{y}}\right)=\operatorname{det}\left(\begin{array}{ccc}
\frac{\partial x_{1}}{\partial y_{1}} & \cdots & \frac{\partial x_{n}}{\partial y_{1}} \\
\vdots & \ddots & \vdots \\
\frac{\partial x_{1}}{\partial y_{n}} & \cdots & \frac{\partial x_{n}}{\partial y_{n}}
\end{array}\right)
$$

which is assumed to be different from zero.

As we will see later, the analysis of Cases I-3, II-3, and III-3-6 requires the computation of the PDF of the sum and product of two continuous RVs which turns out by the application of Theorem 4 in its two-dimensional version. Thus, for the sake of clarity in the exposition, we specialize Theorem 4 in this significant case.

Theorem 5 (RVT technique: two-dimensional version). Let $\mathbf{X}=\left(X_{1}, X_{2}\right)$ be a two-dimensional $R V$ with joint PDF $f_{X_{1}, X_{2}}\left(x_{1}, x_{2}\right)$. Let

$$
\begin{aligned}
& y_{1}=r_{1}\left(x_{1}, x_{2}\right), \\
& y_{2}=r_{2}\left(x_{1}, x_{2}\right),
\end{aligned}
$$

be a one-to-one deterministic map from $\mathbb{R}^{2}$ to $\mathbb{R}^{2}$; that is, there exists its inverse transformation:

$$
\begin{aligned}
& x_{1}=s_{1}\left(y_{1}, y_{2}\right), \\
& x_{2}=s_{2}\left(y_{1}, y_{2}\right),
\end{aligned}
$$

on the range of the map (12). Let one assume that both maps (12) and (13) are continuous. Let further assume that the following partial derivatives

$$
\frac{\partial x_{1}}{\partial y_{1}}, \quad \frac{\partial x_{1}}{\partial y_{2}}, \quad \frac{\partial x_{2}}{\partial y_{1}}, \quad \frac{\partial x_{2}}{\partial y_{2}}
$$

exist and are continuous and the Jacobian $J_{2}$ of the inverse map satisfies

$$
J_{2}=\operatorname{det}\left(\begin{array}{ll}
\frac{\partial x_{1}}{\partial y_{1}} & \frac{\partial x_{2}}{\partial y_{1}} \\
\frac{\partial x_{1}}{\partial y_{2}} & \frac{\partial x_{2}}{\partial y_{2}}
\end{array}\right) \neq 0
$$

on the range of the transformation (12). Then, the joint PDF $f_{Y_{1}, Y_{2}}\left(y_{1}, y_{2}\right)$ of the two-dimensional $R V \mathbf{Y}=\left(Y_{1}, Y_{2}\right)=$ $\left(r_{1}\left(X_{1}, X_{2}\right), r_{2}\left(X_{1}, X_{2}\right)\right)$ is given by

$$
f_{Y_{1}, Y_{2}}\left(y_{1}, y_{2}\right)=f_{X_{1}, X_{2}}\left(s_{1}\left(y_{1}, y_{2}\right), s_{2}\left(y_{1}, y_{2}\right)\right)\left|J_{2}\right| \text {. }
$$

Next, we apply Theorem 5 in the particular case that transformation $r_{1}$ only depends on variable $x_{1}$ and $r_{2}$ only depends on variable $x_{2}$. As it will be seen later, this result will be crucial in further applications.

Proposition 6. Let $\mathbf{X}=\left(X_{1}, X_{2}\right)$ be a two-dimensional $R V$ with joint PDF $f_{X_{1}, X_{2}}\left(x_{1}, x_{2}\right)$. Let

$$
\begin{aligned}
& y_{1}=r_{1}\left(x_{1}\right), \\
& y_{2}=r_{2}\left(x_{2}\right),
\end{aligned}
$$

be a one-to-one deterministic map from $\mathbb{R}^{2}$ to $\mathbb{R}^{2}$; that is, there exists its inverse transformation:

$$
\begin{aligned}
& x_{1}=s_{1}\left(y_{1}\right), \\
& x_{2}=s_{2}\left(y_{2}\right),
\end{aligned}
$$

on the range of the map (17). Let one assume that both maps (17) and (18) are continuous and the two following derivatives that exist are continuous and satisfy

$$
\frac{d x_{1}}{d y_{1}} \frac{d x_{2}}{d y_{2}} \neq 0
$$

on the range of the transformation (17). Then, the joint PDF $f_{Y_{1}, Y_{2}}\left(y_{1}, y_{2}\right)$ of the two-dimensional $R V \mathbf{Y}=\left(Y_{1}, Y_{2}\right)=$ $\left(r_{1}\left(X_{1}\right), r_{2}\left(X_{2}\right)\right)$ is given by

$$
f_{Y_{1}, Y_{2}}\left(y_{1}, y_{2}\right)=f_{X_{1}, X_{2}}\left(s_{1}\left(y_{1}\right), s_{2}\left(y_{2}\right)\right)\left|\frac{d x_{1}}{d y_{1}} \frac{d x_{2}}{d y_{2}}\right| \text {. }
$$


On the other hand, applying Theorem 5 to $r_{1}\left(x_{1}, x_{2}\right)=$ $x_{1}+x_{2}$ and $r_{2}\left(x_{1}, x_{2}\right)=x_{1}$ (or $\left.r_{2}\left(x_{1}, x_{2}\right)=x_{2}\right)$ and $r_{1}\left(x_{1}, x_{2}\right)=x_{1} x_{2}$ and $r_{2}\left(x_{1}, x_{2}\right)=x_{1}\left(\right.$ or $\left.r_{2}\left(x_{1}, x_{2}\right)=x_{2}\right)$, we obtain the PDF of the sum and product of two continuous RVs, respectively. We state both results in the two following propositions.

Proposition 7 (RVT technique: sum of two continuous RVs). Let $\left(X_{1}, X_{2}\right)$ be a continuous random vector with joint PDF $f_{X_{1}, X_{2}}\left(x_{1}, x_{2}\right)$ and respective domains $D_{X_{1}}=\left\{x_{1}: x_{1,1} \leq x_{1} \leq\right.$ $\left.x_{1,2}\right\}$ and $D_{X_{2}}=\left\{x_{2}: x_{2,1} \leq x_{2} \leq x_{2,2}\right\}$. Then the PDF $f_{Y_{1}}\left(y_{1}\right)$ of their sum $Y_{1}=X_{1}+X_{2}$ is given by

$$
\begin{aligned}
& f_{Y_{1}}\left(y_{1}\right)=\int_{x_{1,1}}^{x_{1,2}} f_{X_{1}, X_{2}}\left(x_{1}, y_{1}-x_{1}\right) d x_{1}, \\
& y_{1,1}=x_{1,1}+x_{2,1} \leq y_{1} \leq x_{1,2}+x_{2,2}=y_{1,2},
\end{aligned}
$$

or, equivalent, by

$$
\begin{aligned}
& f_{Y_{1}}\left(y_{1}\right)=\int_{x_{2,1}}^{x_{2,2}} f_{X_{1}, X_{2}}\left(y_{1}-x_{2}, x_{2}\right) d x_{2}, \\
& y_{1,1}=x_{1,1}+x_{2,1} \leq y_{1} \leq x_{1,2}+x_{2,2}=y_{1,2} .
\end{aligned}
$$

If $X_{1}$ and $X_{2}$ are independent continuous $R V s$, since $f_{X_{1}, X_{2}}\left(x_{1}, x_{2}\right)=f_{X_{1}}\left(x_{1}\right) f_{X_{2}}\left(x_{2}\right)$, where $f_{X_{i}}\left(x_{i}\right)$ denotes the PDF of $X_{i}, i=1,2,(21)$ and (22) inform one that the PDF of the sum of two independent continuous $R V$ s is just the convolution of their respective PDFs:

$$
\begin{aligned}
& f_{Y_{1}}\left(y_{1}\right)=\int_{x_{1,1}}^{x_{1,2}} f_{X_{1}}\left(x_{1}\right) f_{X_{2}}\left(y_{1}-x_{1}\right) d x_{1}, \\
& \text { or } f_{Y_{1}}\left(y_{1}\right)=\int_{x_{2,1}}^{x_{2,2}} f_{X_{1}}\left(y_{1}-x_{2}\right) f_{X_{2}}\left(x_{2}\right) d x_{2} .
\end{aligned}
$$

Proposition 8 (RVT technique: product of two continuous $\mathrm{RVs})$. Let $\left(X_{1}, X_{2}\right)$ be a continuous random vector with joint PDF $f_{X_{1}, X_{2}}\left(x_{1}, x_{2}\right)$ with respective domains $D_{X_{1}}=\left\{x_{1} \neq 0\right.$ : $\left.x_{1,1} \leq x_{1} \leq x_{1,2}\right\}$ and $D_{X_{2}}=\left\{x_{2}: x_{2,1} \leq x_{2} \leq x_{2,2}\right\}$. Then the PDF $f_{Y_{1}}\left(y_{1}\right)$ of their product $Y_{1}=X_{1} X_{2}$ is given by

$$
f_{Y_{1}}\left(y_{1}\right)=\int_{x_{1,1}}^{x_{1,2}} f_{X_{1}, X_{2}}\left(x_{1}, \frac{y_{1}}{x_{1}}\right) \frac{1}{\left|x_{1}\right|} d x_{1} .
$$

Equivalently, if $D_{X_{1}}=\left\{x_{1}: x_{1,1} \leq x_{1} \leq x_{1,2}\right\}$ and $D_{X_{2}}=$ $\left\{x_{2} \neq 0: x_{2,1} \leq x_{2} \leq x_{2,2}\right\}$, then

$$
f_{Y_{1}}\left(y_{1}\right)=\int_{x_{2,1}}^{x_{2,2}} f_{X_{1}, X_{2}}\left(\frac{y_{1}}{x_{2}}, x_{2}\right) \frac{1}{\left|x_{2}\right|} d x_{2} .
$$

If $X_{1}$ and $X_{2}$ are independent continuous RVs with PDF's $f_{X_{1}}\left(x_{1}\right)$ and $f_{X_{2}}\left(x_{2}\right)$, respectively, then (24) and (25) become

$$
\begin{aligned}
& f_{Y_{1}}\left(y_{1}\right)=\int_{x_{1,1}}^{x_{1,2}} f_{X_{1}}\left(x_{1}\right) f_{X_{2}}\left(\frac{y_{1}}{x_{1}}\right) \frac{1}{\left|x_{1}\right|} d x_{1}, \\
& \text { or } f_{Y_{1}}\left(y_{1}\right)=\int_{x_{2,1}}^{x_{2,2}} f_{X_{1}}\left(\frac{y_{1}}{x_{2}}\right) f_{X_{2}}\left(x_{2}\right) \frac{1}{\left|x_{2}\right|} d x_{2},
\end{aligned}
$$

As usual we have not specified the domain of variation of $y_{1}$ in (24) and (25) since it is cumbersome. However, later we will detail it in some illustrative cases where it appears (see for instance Case III.6).

We close this section by extending Proposition 7 for the case of three terms since it will be required to deal with Case III.7. This result comes directly from the application of Theorem 4.

Proposition 9 (RVT technique: sum of three continuous RVs). Let $\left(X_{1}, X_{2}, X_{3}\right)$ be a continuous random vector with joint PDF $f_{X_{1}, X_{2}, X_{3}}\left(x_{1}, x_{2}, x_{3}\right)$ and respective domains $D_{X_{1}}=$ $\left\{x_{1}: x_{1,1} \leq x_{1} \leq x_{1,2}\right\}, D_{X_{2}}=\left\{x_{2}: x_{2,1} \leq x_{2} \leq x_{2,2}\right\}$, and $D_{X_{3}}=\left\{x_{3}: x_{3,1} \leq x_{3} \leq x_{3,2}\right\}$. Then the PDF $f_{Y_{1}}\left(y_{1}\right)$ of their sum $Y_{1}=X_{1}+X_{2}+X_{3}$ is given by

$$
\begin{aligned}
& f_{Y_{1}}\left(y_{1}\right)=\int_{x_{3,1}}^{x_{3,2}} \int_{x_{2,1}}^{x_{2,2}} f_{X_{1}, X_{2}, X_{3}}\left(y_{1}-x_{2}-x_{3}, x_{2}, x_{3}\right) d x_{2} d x_{3}, \\
& y_{1,1}=x_{1,1}+x_{2,1}+x_{3,1} \leq y_{1} \leq x_{1,2}+x_{2,2}+x_{3,2}=y_{1,2},
\end{aligned}
$$

or, equivalent, by

$$
f_{Y_{1}}\left(y_{1}\right)=\int_{x_{3,1}}^{x_{3,2}} \int_{x_{1,1}}^{x_{1,2}} f_{X_{1}, X_{2}, X_{3}}\left(x_{1}, y_{1}-x_{1}-x_{3}, x_{3}\right) d x_{1} d x_{3},
$$

or

$$
f_{Y_{1}}\left(y_{1}\right)=\int_{x_{2,1}}^{x_{2,2}} \int_{x_{1,1}}^{x_{1,2}} f_{X_{1}, X_{2}, X_{3}}\left(x_{1}, x_{2}, y_{1}-x_{1}-x_{2}\right) d x_{1} d x_{2} .
$$

\section{Case Study: Initial Value Problem (I)}

This section is devoted to obtain the 1-PDF $f_{1}(z, t)$ of the solution SP $Z(t)$ to the IVP (I) in each of the three cases listed in Table 1. Notice that $Z(t)$ has the following expression:

$$
Z(t)=Z_{0} \mathrm{e}^{A\left(t-t_{0}\right)}, \quad t \geq t_{0} .
$$

3.1. Case I.1: $Z_{0}$ Is a Random Variable. As we pointed out previously for the sake of clarity in the presentation, we rewrite (30) by distinguishing the deterministic character of parameter $a$ (which is written with a lower case letter):

$$
Z(t)=Z_{0} \mathrm{e}^{a\left(t-t_{0}\right)}, \quad t \geq t_{0} .
$$

Next, we first fix $t: t \geq t_{0}$ and denote $Z=Z(t)$. Then we apply Proposition 2 to

$$
\alpha=\mathrm{e}^{a\left(t-t_{0}\right)}>0, \quad \beta=0, \quad X=Z_{0}, \quad Y=Z .
$$

Then, taking into account that the domain of $\mathrm{RV} Z_{0}$ is given by (2), one gets

$$
f_{1}(z, t)=\mathrm{e}^{-a\left(t-t_{0}\right)} f_{Z_{0}}\left(z \mathrm{e}^{-a\left(t-t_{0}\right)}\right), \quad z_{1} \leq z \leq z_{2}, t \geq t_{0},
$$

respectively. 


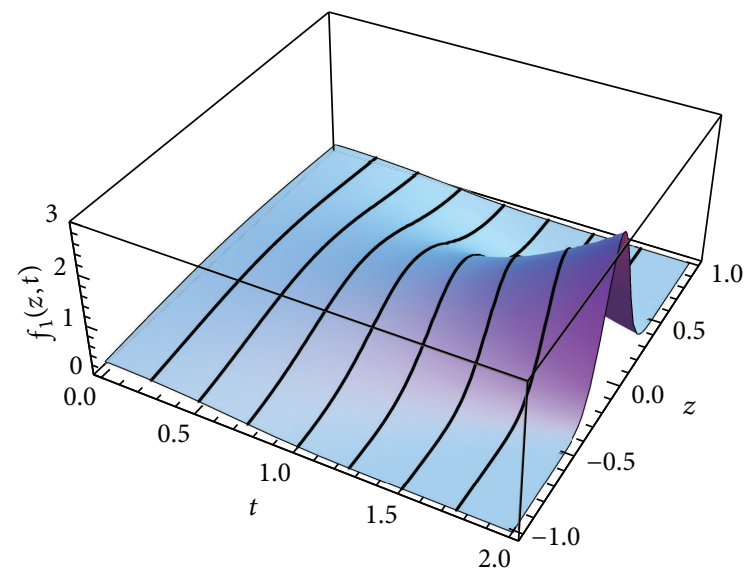

Figure 1: Plot of $f_{1}(z, t)$ given by (35) in Example 10 at different values of $t=\{0,0.25,0.5,0.75, \ldots, 2\}$ (corresponding to the solid lines) in the case that $Z_{0} \sim N\left(\mu=0 ; \sigma^{2}=1\right), t_{0}=0$, and $a=-1$.

where

$$
z_{1}=z_{0,1} \mathrm{e}^{a\left(t-t_{0}\right)}, \quad z_{2}=z_{0,2} \mathrm{e}^{a\left(t-t_{0}\right)} .
$$

We illustrate the previous development in the following example where $Z_{0}$ is assumed to be a standard continuous $\mathrm{RV}$, although further distributions, not necessarily standard, could be considered.

Example 10. Let us assume that $Z_{0}$ has a Gaussian distribution, $Z_{0} \sim N\left(\mu ; \sigma^{2}\right), \mu \in \mathbb{R}$, and $\sigma^{2}>0$. Therefore, according to (33)-(34), the 1-PDF of $Z(t)$ is given by

$$
\begin{array}{r}
f_{1}(z, t)=\frac{1}{\sqrt{2 \pi \sigma^{2}}} \mathrm{e}^{-\left(a\left(t-t_{0}\right)+\left(1 / 2 \sigma^{2}\right)\left(z \mathrm{e}^{-a\left(t-t_{0}\right)}-\mu\right)^{2}\right)}, \\
-\infty<z<+\infty, t \geq t_{0} .
\end{array}
$$

For each $t \geq t_{0}$, the domain of $z$ has been determined taking into account in (34) that in this case $z_{0,1}=-\infty$ and $z_{0,2}=$ $+\infty$. It can be checked that $f_{1}(z, t)$ is a PDF for each $t \geq t_{0}$. Figure 1 shows $f_{1}(z, t)$ at different values of $t$ in the particular case that $t_{0}=0, a=-1$ and $Z_{0} \sim N(0 ; 1)$, so $\mu=0$ and $\sigma=1$.

3.2. Case I.2: A Is a Random Variable. First, we rewrite (30) by highlighting the deterministic character of the initial condition $z_{0}$ :

$$
Z(t)=z_{0} \mathrm{e}^{A\left(t-t_{0}\right)}, \quad t \geq t_{0} .
$$

Next, we fix $t: t>t_{0}$ and denote $Z=Z(t)$. In the first analysis, we will assume that $z_{0} \neq 0$. Then we apply Proposition 3 to

$$
\begin{gathered}
\alpha=z_{0} \neq 0, \quad \beta=t-t_{0}, \quad X=A, \\
\gamma=0, \quad Y=Z .
\end{gathered}
$$

Then, taking into account that $z / z_{0}=\mathrm{e}^{a\left(t-t_{0}\right)}>0$ and the domain of RV $A$ is given by (2), one gets

$$
\begin{array}{r}
f_{1}(z, t)=\frac{1}{\left(t-t_{0}\right)|z|} f_{A}\left(\frac{1}{t-t_{0}} \ln \left(\frac{z}{z_{0}}\right)\right), \\
z_{1} \leq z \leq z_{2}, t>t_{0},
\end{array}
$$

where

$$
\begin{aligned}
& z_{1}=z_{0} \mathrm{e}^{a_{1}\left(t-t_{0}\right)}, \quad z_{2}=z_{0} \mathrm{e}^{a_{2}\left(t-t_{0}\right)}, \quad \text { if } z_{0}>0, \\
& z_{1}=z_{0} \mathrm{e}^{a_{2}\left(t-t_{0}\right)}, \quad z_{2}=z_{0} \mathrm{e}^{a_{1}\left(t-t_{0}\right)}, \quad \text { if } z_{0}<0 .
\end{aligned}
$$

According to (2), if $z_{0}=0$, then

$$
f_{1}(z, t)=\delta(z), \quad-\infty<z<\infty, \forall t>t_{0} .
$$

For $t=t_{0}$, from (36) $Z(t)=Z\left(t_{0}\right)=z_{0}$, which is a deterministic initial condition. Then its 1-PDF can be written through the Dirac delta function as follows:

$$
f_{1}\left(z, t_{0}\right)=\delta\left(z-z_{0}\right), \quad-\infty<z<\infty .
$$

In the example below, we illustrate the previous development in the case where $z_{0}>0$.

Example 11. Let us assume that $A$ has a beta distribution, $A$ $\operatorname{Be}(\alpha ; \beta), \alpha, \beta>0$, and $z_{0}>0$. Therefore, according to (38)(39), the 1-PDF of $Z(t)$ is given by

$$
\begin{gathered}
f_{1}(z, t)=\frac{1}{B(\alpha, \beta)|z|}\left(\frac{1}{t-t_{0}}\right)^{\alpha}\left(\ln \left(\frac{z}{z_{0}}\right)\right)^{\alpha-1} \\
\times\left(1-\frac{1}{t-t_{0}} \ln \left(\frac{z}{z_{0}}\right)\right)^{\beta-1}, \\
z_{0} \leq z \leq z_{0} \mathrm{e}^{t-t_{0}}, t>t_{0},
\end{gathered}
$$

where $B(\alpha, \beta)$ denotes the beta deterministic special function. Since $z=z_{0} \mathrm{e}^{a\left(t-t_{0}\right)}$ and $0 \leq a \leq 1$, it is guaranteed that $0 \leq$ $\left(1 /\left(t-t_{0}\right)\right) \ln \left(z / z_{0}\right) \leq 1$. As a consequence, $f_{1}(z, t)$ given by (42) is well defined. For each $t>t_{0}$, the domain of $z$ has been determined taking into account in (39) that in this case $a_{1}=0$ and $a_{2}=1$. It can be checked that $f_{1}(z, t)$ is a PDF for each $t>t_{0}$. Figure 2 shows $f_{1}(z, t)$ at different values of $t$ in the particular case that $A \sim \operatorname{Be}(2 ; 3), t_{0}=0$, and $z_{0}=1$. For $t=0$, according to (41), $f_{1}(z, 0)=\delta(z-1),-\infty<z<\infty$.

3.3. Case I.3: $\left(Z_{0}, A\right)$ Is a Random Vector. Let us denote by $f_{Z_{0}, A}\left(z_{0}, a\right)$ the joint PDF of the random vector $\left(Z_{0}, A\right)$. Now, we rewrite (30) in the following equivalent form:

$$
Z(t)=Z_{1}(t) Z_{2}(t), \quad \text { where }\left\{\begin{array}{l}
Z_{1}(t)=Z_{0}, \\
Z_{2}(t)=\mathrm{e}^{A\left(t-t_{0}\right)} .
\end{array}\right.
$$

In order to apply RVT, we fix $t: t>t_{0}$ and denote $Z_{1}=Z_{1}(t)$, $Z_{2}=Z_{2}(t)$, and $Z=Z_{1} Z_{2}=Z(t)$. To compute the PDF of $Z$, 


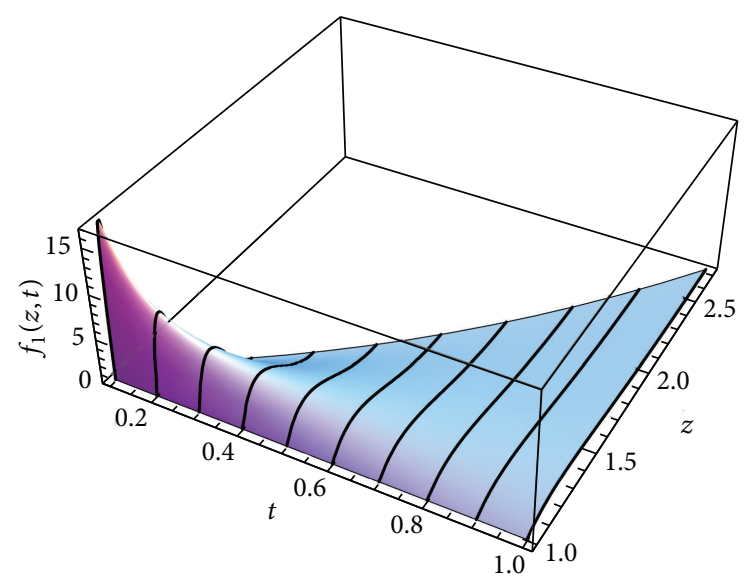

Figure 2: Plot of $f_{1}(z, t)$ given by (42) in Example 11 at different values of $t=\{0.1,0.2,0.3, \ldots, 1\}$ (corresponding to the solid lines) in the case that $A \sim \operatorname{Be}(2 ; 3), t_{0}=0$, and $z_{0}=1$.

first we will determine the joint PDF of $Z_{1}$ and $Z_{2}$ by applying Proposition 6 to

$$
\begin{aligned}
& X_{1}=Z_{0}, \quad X_{2}=A, \quad r_{1}\left(z_{0}\right)=z_{0}, \\
& r_{2}(a)=\mathrm{e}^{a\left(t-t_{0}\right)}, \quad Y_{1}=Z_{1}, \quad Y_{2}=Z_{2}, \\
& s_{1}\left(z_{1}\right)=z_{1}, \quad s_{2}\left(z_{2}\right)=\frac{\ln \left(z_{2}\right)}{t-t_{0}} .
\end{aligned}
$$

Then taking into account

$$
\frac{\mathrm{d} s_{1}\left(z_{1}\right)}{\mathrm{d} z_{1}} \frac{\mathrm{d} s_{2}\left(z_{2}\right)}{\mathrm{d} z_{2}}=\frac{1}{z_{2}\left(t-t_{0}\right)}>0
$$

one gets

$$
\begin{array}{r}
f_{Z_{1}, Z_{2}}\left(z_{1}, z_{2}\right)=\frac{1}{z_{2}\left(t-t_{0}\right)} f_{Z_{0}, A}\left(z_{1}, \frac{\ln \left(z_{2}\right)}{t-t_{0}}\right), \\
z_{0,1} \leq z_{1} \leq z_{0,2}, \mathrm{e}^{a_{1}\left(t-t_{0}\right)} \leq z_{2} \leq \mathrm{e}^{a_{2}\left(t-t_{0}\right)} .
\end{array}
$$

Once the joint PDF of $\left(Z_{1}, Z_{2}\right)$ has been determined, the computation of the PDF of $Z=Z_{1} Z_{2}$ follows directly by applying Proposition 8. Indeed, as $Z_{2}=\mathrm{e}^{A\left(t-t_{0}\right)} \neq 0$, we will apply formula (25) to $X_{1}=Z_{1}=Z_{0}, X_{2}=Z_{2}=\mathrm{e}^{A\left(t-t_{0}\right)}>0$ and $Y_{1}=Z=Z_{1} Z_{2}$. This yields

$$
f_{1}(z, t)=f_{Z}(z)=\int_{z_{2,1}}^{z_{2,2}} f_{Z_{1}, Z_{2}}\left(\frac{z}{z_{2}}, z_{2}\right) \frac{1}{z_{2}} \mathrm{~d} z_{2},
$$

or equivalently by using (46):

$$
\begin{array}{r}
f_{1}(z, t)=\int_{z_{2,1}}^{z_{2,2}} f_{Z_{0}, A}\left(\frac{z}{z_{2}}, \frac{\ln \left(z_{2}\right)}{t-t_{0}}\right) \\
z_{1} \leq z \leq z_{2}, t>t_{0},
\end{array}
$$

where

$$
\begin{gathered}
z_{2,1}=\mathrm{e}^{a_{1}\left(t-t_{0}\right)}, \quad z_{2,2}=\mathrm{e}^{a_{2}\left(t-t_{0}\right)}, \\
z_{1}=z_{0,1} \mathrm{e}^{a_{1}\left(t-t_{0}\right)}, \quad z_{2}=z_{0,2} \mathrm{e}^{a_{2}\left(t-t_{0}\right)}, \quad \text { if } z_{0,1}>0, \\
z_{1}=z_{0,1} \mathrm{e}^{a_{2}\left(t-t_{0}\right)}, \quad z_{2}=z_{0,2} \mathrm{e}^{a_{2}\left(t-t_{0}\right)}, \quad \text { if } z_{0,1} z_{0,2} \leq 0, \\
z_{1}=z_{0,1} \mathrm{e}^{a_{2}\left(t-t_{0}\right)}, \quad z_{2}=z_{0,2} \mathrm{e}^{a_{1}\left(t-t_{0}\right)}, \quad \text { if } z_{0,2}<0 .
\end{gathered}
$$

Notice that if $t=t_{0}$, then $Z(t)=Z\left(t_{0}\right)=Z_{0}$ and $f_{1}(z, t)$ is just the PDF of RV $Z_{0}$, which is easily obtained from the datum $f_{Z_{0}, A}\left(z_{0}, a\right)$ as the following marginal distribution:

$$
\begin{aligned}
f_{1}\left(z_{0}, t_{0}\right) & =f_{Z_{0}}\left(z_{0}\right) \\
& =\int_{a_{1}}^{a_{2}} f_{Z_{0}, A}\left(z_{0}, a\right) \mathrm{d} a, \quad z_{0,1} \leq z_{0} \leq z_{0,2} .
\end{aligned}
$$

Example 12. Let $\left(Z_{0}, A\right)$ be a two-dimensional RV whose PDF is defined by

$$
f_{Z_{0}, A}\left(z_{0}, a\right)= \begin{cases}4 a z_{0} & \text { if } 0<z_{0}, a<1 \\ 0 & \text { otherwise. }\end{cases}
$$

Notice that we are implicitly assuming independence between $Z_{0}$ and $A$ since the joint $\operatorname{PDF} f_{Z_{0}, A}\left(z_{0}, a\right)$ factorizes as the product of the marginal PDFs $f_{Z_{0}}\left(z_{0}\right)=2 z_{0}$, $0<z_{0}<1$, and $f_{A}(a)=2 a, 0<a<1$. Then, taking into account (48)-(50) and (52), the 1-PDF of $Z(t)$ is given by

$$
\begin{aligned}
& f_{1}(z, t) \\
& =\left\{\begin{array}{ll}
\frac{4 z}{\left(t-t_{0}\right)^{2}} \int_{1}^{\mathrm{e}^{t-t_{0}}} \frac{\ln \left(z_{2}\right)}{\left(z_{2}\right)^{3}} \mathrm{~d} z_{2} & \text { if } 0 \leq z \leq 1, \\
\frac{4 z}{\left(t-t_{0}\right)^{2}} \int_{z}^{\mathrm{e}^{t-t_{0}}} \frac{\ln \left(z_{2}\right)}{\left(z_{2}\right)^{3}} \mathrm{~d} z_{2} & \text { if } 1 \leq z \leq \mathrm{e}^{t-t_{0}},
\end{array} \quad t>t_{0} .\right.
\end{aligned}
$$

For the sake of clarity in the graphical representation of $f_{1}(z, t)$, Figure 3 shows two equivalent plots of $f_{1}(z, t)$ in the case that $t_{0}=0$. Notice that the plot on the left side is the resultant surface where some 1-PDF $f_{1}(z, t)$ have been highlighted for different fixed times, whereas these 1-PDFs have been represented in these fixed times on the right side. As the two integrals appearing into the expression (53) can be computed explicitly, an equivalent expression to $f_{1}(z, t)$ with $t_{0}=0$ is given by

$$
\begin{aligned}
& f_{1}(z, t) \\
& =\left\{\begin{array}{ll}
\frac{z}{t^{2}} \mathrm{e}^{-2 t}\left(-1+\mathrm{e}^{2 t}-2 t\right) & \text { if } 0 \leq z \leq 1, \\
\frac{z}{t^{2}}\left(-\mathrm{e}^{-2 t}(1+2 t)+\frac{1+2 \ln (z)}{z^{2}}\right) & \text { if } 1 \leq z \leq \mathrm{e}^{t}, t>0 .
\end{array} t\right.
\end{aligned}
$$




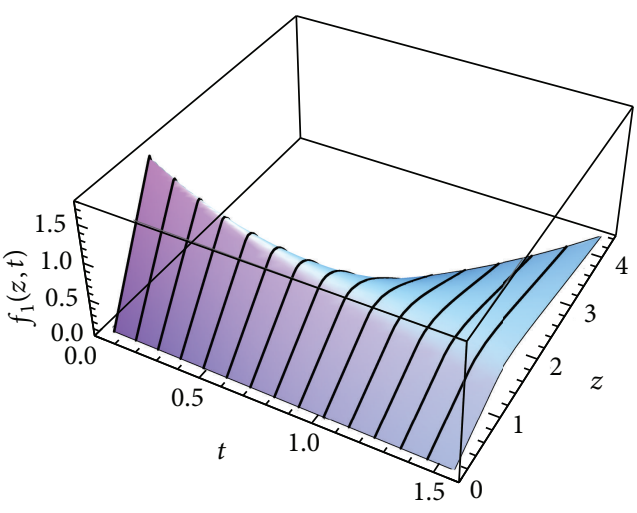

(a)

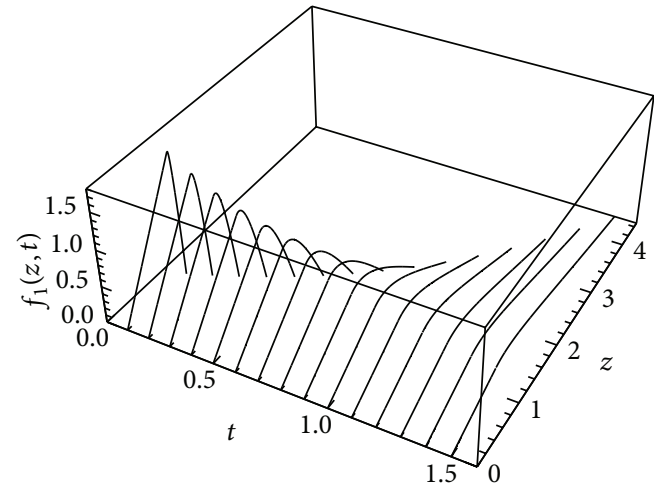

(b)

Figure 3: Plots of $f_{1}(z, t)$ given by (54) in Example 12 at different values of $t=\{0.1,0.2,0.3, \ldots, 1.5\}$ (corresponding to the solid lines in both plots) in the case that $\left(Z_{0}, A\right)$ is a two-dimensional RV whose PDF is given by (52) and $t_{0}=0$.

For $t=0$,

$$
\begin{aligned}
f_{1}\left(z_{0}, 0\right) & =f_{Z_{0}}\left(z_{0}\right) \\
& =\int_{0}^{1} 4 a z_{0} \mathrm{~d} a=2 z_{0}, \quad z_{0,1}=0<z<1=z_{0,2} .
\end{aligned}
$$

\section{Case Study: Initial Value Problem (II)}

This section is addressed to determine the 1-PDF $f_{1}(z, t)$ of the solution SP $Z(t)$ to the IVP (II) in each of the three cases listed in Table 1 . Notice that $Z(t)$ has the following expression:

$$
Z(t)=Z_{0}+B\left(t-t_{0}\right), \quad t \geq t_{0}
$$

4.1. Case II.1: $Z_{0}$ Is a Random Variable. As we did in Case I.1, for the sake of clarity in the presentation, we rewrite (56) by emphasizing the deterministic character of parameter $b$ (which is written with a lower case letter):

$$
Z(t)=Z_{0}+b\left(t-t_{0}\right), \quad t \geq t_{0} .
$$

Next, we fix $t: t \geq t_{0}$ and denote $Z=Z(t)$. Then we apply Proposition 2 to

$$
\alpha=1>0, \quad \beta=b\left(t-t_{0}\right), \quad X=Z_{0}, \quad Y=Z \text {. }
$$

Taking into account that the domain of $\mathrm{RV} Z_{0}$ is given by (2), one gets

$$
f_{1}(z, t)=f_{Z_{0}}\left(z-b\left(t-t_{0}\right)\right), \quad z_{1} \leq z \leq z_{2}, t \geq t_{0},
$$

where

$$
z_{1}=z_{0,1}+b\left(t-t_{0}\right), \quad z_{2}=z_{0,2}+b\left(t-t_{0}\right) .
$$

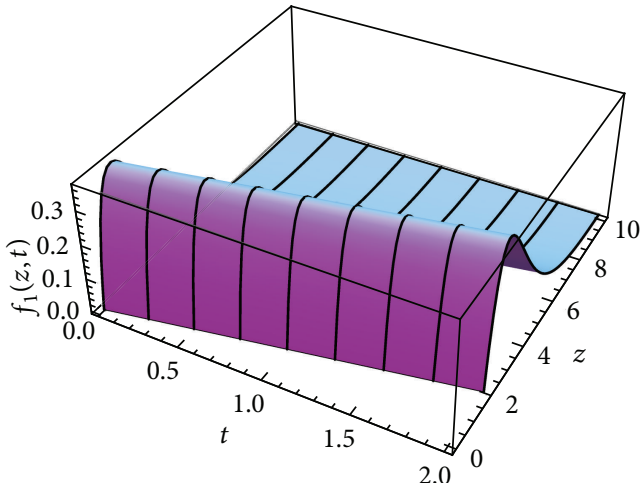

Figure 4: Plot of $f_{1}(z, t)$ given by (61) in Example 13 at different values of $t=\{0,0.25,0.5, \ldots, 2\}$ (corresponding to the solid lines) in the case that $\mathrm{Ga}(2 ; 1), b=1$, and $t_{0}=0$.

Example 13. Let us assume that $Z_{0}$ has a gamma distribution, $Z_{0} \sim \mathrm{Ga}(\alpha ; \beta), \alpha, \beta>0$. Therefore, according to (59)-(60), the 1-PDF of $Z(t)$ is given by:

$$
\begin{array}{r}
f_{1}(z, t)=\frac{1}{\beta^{\alpha} \Gamma(\alpha)}\left(z-b\left(t-t_{0}\right)\right)^{\alpha-1} \mathrm{e}^{-\left(z-b\left(t-t_{0}\right)\right) / \beta}, \\
b\left(t-t_{0}\right) \leq z<+\infty, t \geq t_{0},
\end{array}
$$

where $\Gamma(\alpha)$ denotes the deterministic gamma special function. For each $t \geq t_{0}$, the domain of $z$ has been determined taking into account in (60) that, in this case, $z_{0,1}=0$ and $z_{0,2}=+\infty$. It can be checked that $f_{1}(z, t)$ is a PDF for each $t \geq t_{0}$. Figure 4 shows $f_{1}(z, t)$ at different values of $t$ in the particular case that $Z_{0} \sim \mathrm{Ga}(2 ; 1), b=1$, and $t_{0}=0$.

4.2. Case II.2: B Is a Random Variable. First, we rewrite (56) by highlighting the deterministic character of the initial condition $z_{0}$ :

$$
Z(t)=z_{0}+B\left(t-t_{0}\right), \quad t \geq t_{0} .
$$




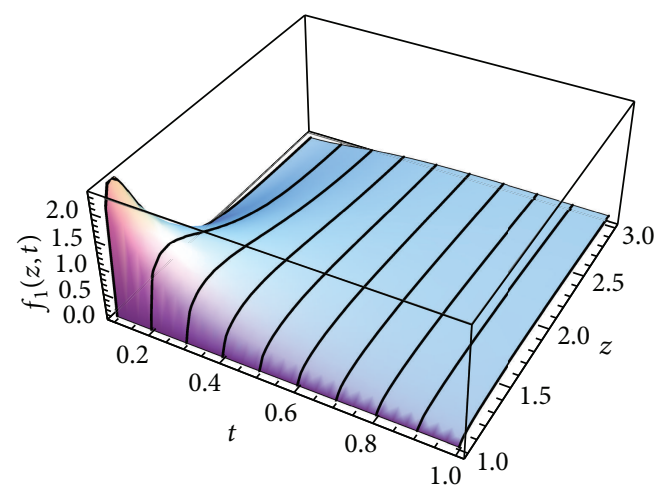

Figure 5: Plot of $f_{1}(z, t)$ given by (67) in Example 14 at different values of $t=\{0.1,0.2,0.3, \ldots, 1\}$ (corresponding to the solid lines) in the case that $B \sim \chi^{2}(3), t_{0}=0$, and $z_{0}=1$.

Next, we fix $t: t>t_{0}$ and denote $Z=Z(t)$. Then we apply Proposition 2 to

$$
\alpha=t-t_{0}>0, \quad \beta=z_{0}, \quad X=B, \quad Y=Z .
$$

This yields

$$
f_{1}(z, t)=\frac{1}{\left(t-t_{0}\right)} f_{B}\left(\frac{z-z_{0}}{t-t_{0}}\right), \quad z_{1} \leq z \leq z_{2}, t>t_{0},
$$

where

$$
z_{1}=z_{0}+b_{1}\left(t-t_{0}\right), \quad z_{2}=z_{0}+b_{2}\left(t-t_{0}\right) .
$$

For $t=t_{0}$, as it also occurred in Case I.2, $Z(t)=Z\left(t_{0}\right)=z_{0}$ and therefore

$$
f_{1}\left(z, t_{0}\right)=\delta\left(z-z_{0}\right), \quad-\infty<z<\infty .
$$

Example 14. Let us assume that $B$ has a $\chi^{2}$-distribution with $v$ degrees of freedom, $B \sim \chi^{2}(v)$, and $v>0$. Therefore, according to (64)-(65), the 1-PDF of $Z(t)$ is given by

$$
\begin{aligned}
f_{1}(z, t)= & \frac{1}{\left(t-t_{0}\right)} \frac{1}{2^{v / 2}} \frac{1}{\Gamma(\nu / 2)}\left(\frac{z-z_{0}}{t-t_{0}}\right)^{(v / 2)-1} \\
& \times \mathrm{e}^{-\left(z-z_{0}\right) / 2\left(t-t_{0}\right)}, \quad z_{0} \leq z<\infty, t>t_{0} .
\end{aligned}
$$

For each $t: t>t_{0}$, the domain of $z$ has been determined taking into account in (65) that in this case $b_{1}=0$ and $b_{2}=\infty$. It can be checked that $f_{1}(z, t)$ is a PDF for each $t>t_{0}$. Figure 5 shows $f_{1}(z, t)$ at different values of $t$ in the particular case that $B \sim \chi^{2}(3), t_{0}=0$, and $z_{0}=1$. For $t=0$, according to (66), $f_{1}(z, 0)=\delta(z-1),-\infty<z<\infty$.

4.3. Case II.3: $\left(Z_{0}, B\right)$ Is a Random Vector. We will denote by $f_{Z_{0}, B}\left(z_{0}, b\right)$ the joint PDF of continuous RVs $Z_{0}$ and $B$. Let us rewrite (56) in the following equivalent form:

$$
Z(t)=Z_{1}(t)+Z_{2}(t), \quad \text { where }\left\{\begin{array}{l}
Z_{1}(t)=Z_{0} \\
Z_{2}(t)=B\left(t-t_{0}\right)
\end{array}\right.
$$

In order to compute the 1-PDF of $Z(t), f_{1}(z, t)$, we first fix $t: t>t_{0}$ and consider the continuous RVs $Z_{1}=Z_{1}(t)$, $Z_{2}=Z_{2}(t)$, and $Z=Z_{1}+Z_{2}=Z(t)$. Then, we will apply Propositions 6 and 7. Indeed, in a first step we compute the joint PDF $f_{Z_{1}, Z_{2}}\left(z_{1}, z_{2}\right)$ by applying Proposition 6 to

$$
\begin{aligned}
& X_{1}=Z_{0}, \quad X_{2}=B, \quad r_{1}\left(z_{0}\right)=z_{0}, \\
& r_{2}(b)=b\left(t-t_{0}\right), \quad Y_{1}=Z_{1}, \quad Y_{2}=Z_{2}, \\
& s_{1}\left(z_{1}\right)=z_{1}, \quad s_{2}\left(z_{2}\right)=\frac{z_{2}}{t-t_{0}} .
\end{aligned}
$$

Taking into account that

$$
\frac{\mathrm{d} s_{1}\left(z_{1}\right)}{\mathrm{d} z_{1}}=1>0, \quad \frac{\mathrm{d} s_{2}\left(z_{2}\right)}{\mathrm{d} z_{2}}=\frac{1}{t-t_{0}}>0,
$$

one gets

$$
f_{Z_{1}, Z_{2}}\left(z_{1}, z_{2}\right)=\frac{1}{t-t_{0}} f_{Z_{0}, B}\left(z_{1}, \frac{z_{2}}{t-t_{0}}\right),
$$

where $z_{1}$ and $z_{2}$ lie on

$$
\begin{aligned}
& z_{1,1}=z_{0,1} \leq z_{1} \leq z_{0,2}=z_{1,2}, \\
& z_{2,1}=b_{1}\left(t-t_{0}\right) \leq z_{2} \leq b_{2}\left(t-t_{0}\right)=z_{2,2} .
\end{aligned}
$$

Finally, we apply Proposition 7 using the following identification: $X_{1}=Z_{1}, X_{2}=Z_{2}$, and $Y_{1}=Z$. This yields

$$
f_{1}(z, t)=\int_{z_{1,1}}^{z_{1,2}} f_{Z_{1}, Z_{2}}\left(z_{1}, z-z_{1}\right) \mathrm{d} z_{1},
$$

or more explicitly, using that $Z_{1}=Z_{0}$ and (71),

$$
\begin{array}{r}
f_{1}(z, t)=\frac{1}{t-t_{0}} \int_{z_{0,1}}^{z_{0,2}} f_{Z_{0}, B}\left(z_{0}, \frac{z-z_{0}}{t-t_{0}}\right) \mathrm{d} z_{0}, \\
z_{1} \leq z \leq z_{2}, t>t_{0},
\end{array}
$$

where

$$
z_{1}=z_{0,1}+b_{1}\left(t-t_{0}\right) \leq z \leq z_{0,2}+b_{2}\left(t-t_{0}\right)=z_{2} .
$$

If $t=t_{0}$, then $Z(t)=Z\left(t_{0}\right)=Z_{0}$ and, similar to Case I.3, $f_{1}(z, t)$ is just the PDF of RV $Z_{0}$, which is easily obtained from the datum $f_{Z_{0}, B}\left(z_{0}, b\right)$ as the following marginal distribution:

$$
\begin{aligned}
f_{1}\left(z_{0}, t_{0}\right) & =f_{Z_{0}}\left(z_{0}\right) \\
& =\int_{b_{1}}^{b_{2}} f_{Z_{0}, B}\left(z_{0}, b\right) \mathrm{d} b, \quad z_{0,1} \leq z_{0} \leq z_{0,2} .
\end{aligned}
$$

Example 15. Let $\left(Z_{0}, B\right)$ be a two-dimensional vector whose joint $\mathrm{PDF}$ is given by

$$
\begin{aligned}
& f_{Z_{0}, B}\left(z_{0}, b\right) \\
& \quad= \begin{cases}\frac{1}{4}+\frac{1}{4}\left(z_{0}\right)^{3} b-\frac{1}{4} z_{0} b^{3} & \text { if }-1<z_{0}<1,-1<b<1, \\
0 & \text { otherwise. }\end{cases}
\end{aligned}
$$




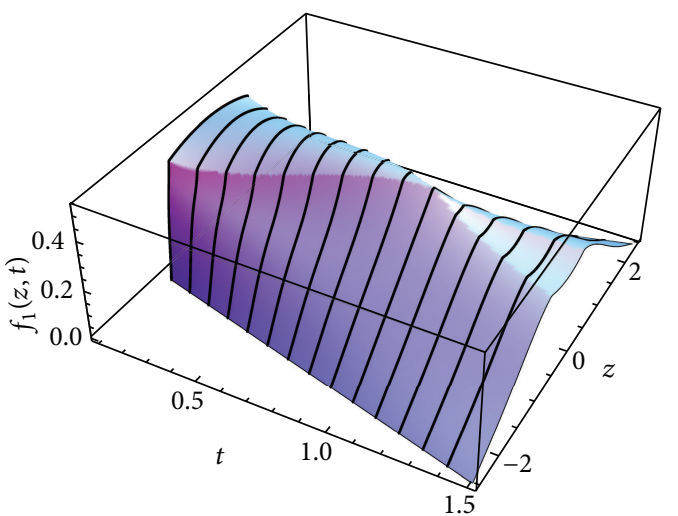

(a)

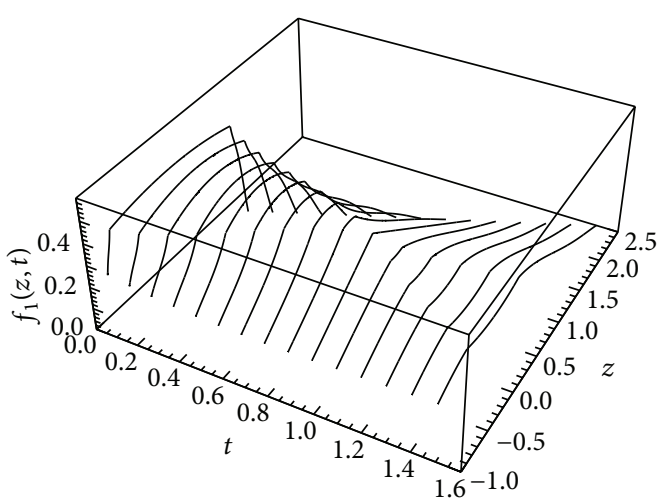

(b)

Figure 6: Plot of $f_{1}(z, t)$ given by (79)-(80) in Example 15 at different values of $t=\{0,0.1,0.2, \ldots, 1.5\}$ (corresponding to the solid lines in both plots) in the case that $\left(Z_{0}, B\right)$ is a two-dimensional RV whose PDF is given by $(77)$ and $t_{0}=0$.

Then, carrying out the involved computations according to (74)-(75), the 1-PDF of $Z(t)$ is given by

$$
\begin{array}{r}
f_{1}(z, t)=\frac{1}{t-t_{0}} \int_{\max \{z-t,-1\}}^{\min \{1, z+t\}}\left(\frac{1}{4}+\frac{1}{4}\left(z_{0}\right)^{3}\left(\frac{z-z_{0}}{t-t_{0}}\right)\right. \\
\left.-\frac{1}{4} z_{0}\left(\frac{z-z_{0}}{t-t_{0}}\right)^{3}\right) \mathrm{d} z_{0} \\
-1-t \leq z \leq 1+t .
\end{array}
$$

Taking into account that $t_{0}=0$, the value of this integral is

$$
f_{1}(z, t)= \begin{cases}f_{1 a}(z, t) & \text { if } t \leq 1,-1-t \leq z \leq-1+t \\ f_{1 b}(z, t) & \text { if } t \leq 1,-1+t \leq z \leq 1-t \\ f_{1 c}(z, t) & \text { if } t \leq 1,1-t \leq z \leq 1+t \\ f_{1 d}(z, t) & \text { if } t \geq 1,-1-t \leq z \leq 1-t \\ f_{1 e}(z, t) & \text { if } t \geq 1,1-t \leq z \leq-1+t \\ f_{1 f}(z, t) & \text { if } t \geq 1,-1+t \leq z \leq 1+t\end{cases}
$$

where

$$
\begin{aligned}
& f_{1 a}(z, t) \\
& =\left(t^{3}+t^{4}+t^{3} z+\frac{1}{20}\left(4 t^{5}+5 t^{4} z-(-4+z)(1+z)^{4}\right)\right. \\
& \left.\quad+\frac{1}{20} t^{2}\left(-4-5 z-(4 t-z)(t+z)^{4}\right)\right) \\
& \times\left(4 t^{4}\right)^{-1}, \\
& \quad f_{1 b}(z, t)=\frac{1}{2}+\frac{t}{10}-\frac{t^{3}}{10}-\frac{t z^{2}}{2},
\end{aligned}
$$

$$
f_{1 c}(z, t)
$$

$$
\begin{aligned}
& =\left(t^{3}+t^{4}-t^{3} z+\frac{1}{20}\left(4 t^{5}-5 t^{4} z+(-1+z)^{4}(4+z)\right)\right. \\
& \left.+\frac{1}{20} t^{2}\left(-4+5 z-(t-z)^{4}(4 t+z)\right)\right) \\
& \times\left(4 t^{4}\right)^{-1} \\
& f_{1 d}(z, t) \\
& =\left(t^{3}+t^{4}+t^{3} z+\frac{1}{20}\left(4 t^{5}+5 t^{4} z-(-4+z)(1+z)^{4}\right)\right. \\
& \left.+\frac{1}{20} t^{2}\left(-4-5 z-(4 t-z)(t+z)^{4}\right)\right) \\
& \times\left(4 t^{4}\right)^{-1} \\
& f_{1 e}(z, t)=\frac{1}{10 t^{4}}-\frac{1}{10 t^{2}}+\frac{1}{2 t}+\frac{z^{2}}{2 t^{4}}, \\
& f_{1 f}(z, t) \\
& =\left(t^{3}+t^{4}-t^{3} z+\frac{1}{20}\left(4 t^{5}-5 t^{4} z+(-1+z)^{4}(4+z)\right)\right. \\
& \left.+\frac{1}{20} t^{2}\left(-4+5 z-(t-z)^{4}(4 t+z)\right)\right) \\
& \times\left(4 t^{4}\right)^{-1} \text {. }
\end{aligned}
$$

As we did in the example of Case I.3 (see Figure 3), for the sake of clarity, Figure 6 shows two equivalent plots of $f_{1}(z, t)$ given by (79)-(80).

\section{Case Study: Initial Value Problem (III)}

This section deals with the computation of the 1-PDF $f_{1}(z, t)$ of the solution SP $Z(t)$ to the IVP (III) in each of the seven 
cases listed in Table 1 . Notice that $Z(t)$ has the following expression:

$$
Z(t)=\left(Z_{0}+\frac{B}{A}\right) \mathrm{e}^{A\left(t-t_{0}\right)}-\frac{B}{A}, \quad t \geq t_{0} .
$$

Throughout the sequent analysis depending on whether $A$ is considered to be a RV (Cases III.3, III.5, III.6, and III.7) or a deterministic constant (Cases III.1, III.2, and III.4), see Table 1 ; we will assume that $\mathbb{P}[\{\omega \in \Omega: A(\omega) \neq 0\}]=1$ or $a \neq 0$, respectively.

5.1. Case III.1: $Z_{0}$ Is a Random Variable. In order to take advantage of the RVT method and to study this case, it is convenient to rewrite (81) in the equivalent form:

$$
Z(t)=\mathrm{e}^{a\left(t-t_{0}\right)} Z_{0}+\frac{b}{a}\left(\mathrm{e}^{a\left(t-t_{0}\right)}-1\right), \quad t \geq t_{0} .
$$

Next, we first fix $t: t \geq t_{0}$ and denote $Z=Z(t)$. Then we apply Proposition 2 to

$$
\begin{aligned}
& \alpha=\mathrm{e}^{a\left(t-t_{0}\right)}>0, \quad \beta=\frac{b}{a}\left(\mathrm{e}^{a\left(t-t_{0}\right)}-1\right), \\
& X=Z_{0}, \quad Y=Z .
\end{aligned}
$$

Then, taking into account that the domain of $\mathrm{RV} Z_{0}$ is given by (2), one gets

$$
\begin{array}{r}
f_{1}(z, t)=\mathrm{e}^{-a\left(t-t_{0}\right)} f_{Z_{0}}\left(\mathrm{e}^{-a\left(t-t_{0}\right)}\left(z+\frac{b}{a}\right)-\frac{b}{a}\right), \\
z_{1} \leq z \leq z_{2}, t \geq t_{0},
\end{array}
$$

where

$$
\begin{aligned}
& z_{1}=z_{0,1} \mathrm{e}^{a\left(t-t_{0}\right)}+\frac{b}{a}\left(\mathrm{e}^{a\left(t-t_{0}\right)}-1\right), \\
& z_{2}=z_{0,2} \mathrm{e}^{a\left(t-t_{0}\right)}+\frac{b}{a}\left(\mathrm{e}^{a\left(t-t_{0}\right)}-1\right)
\end{aligned}
$$

Example 16. Let $Z_{0}$ be an exponential RV, $Z_{0} \sim \operatorname{Exp}(\lambda)$, and let $\lambda>0$. Then, according to (84)-(85), the 1-PDF of $Z(t)$ is given by

$$
\begin{array}{r}
f_{1}(z, t)=\lambda \mathrm{e}^{-\left(a\left(t-t_{0}\right)+\lambda\left((z+(b / a)) \mathrm{e}^{-a\left(t-t_{0}\right)}-(b / a)\right)\right)}, \\
\frac{b}{a}\left(\mathrm{e}^{a\left(t-t_{0}\right)}-1\right) \leq z<+\infty, \quad t \geq t_{0},
\end{array}
$$

where the domain of $z$ has been determined taking into account in (85) that in this case $z_{0,1}=0$ and $z_{0,2}=+\infty$. Again, it can be checked that $f_{1}(z, t)$ is a PDF for each $t \geq t_{0}$. Figure 7 shows $f_{1}(z, t)$ at different values of $t$ in the case that $\lambda=1, t_{0}=0, a=-1$, and $b=1$.

Before closing this case, we provide an example where the usefulness of the 1-PDF $f_{1}(z, t)$ to determine the statistical moments of the solution $Z(t)$ and to compute the probability of sets of interest is shown. To illustrate these applications, we will choose the context of Example 16, although it could be applied to any example throughout this paper.

Example 17. Let us consider the context of Example 16 where the 1-PDF of $Z(t), f_{1}(z, t)$, has been computed (see expression (86)). Then, the statistical moment of order $n$ of $Z(t)$ with respect to the origin can be computed directly in terms of $f_{1}(z, t)$ as follows:

$$
\begin{array}{r}
m_{Z}(t, n)=E\left[(Z(t))^{n}\right]=\int_{b / a\left(\mathrm{e}^{a\left(t-t_{0}\right)}-1\right)}^{+\infty} z^{n} f_{1}(z, t) \mathrm{d} z \\
n=0,1,2, \ldots .
\end{array}
$$

As a consequence, the following expressions for the mean and the variance of $Z(t)$ are obtained:

$$
\begin{aligned}
& \mathrm{E}[Z(t)]=m_{Z}(t, 1)=\frac{-b \lambda+\mathrm{e}^{a\left(t-t_{0}\right)}(a+b \lambda)}{\lambda a}, \\
& \operatorname{Var}[Z(t)]=m_{Z}(t, 2)-\left(m_{Z}(t, 1)\right)^{2}=\frac{\mathrm{e}^{2 a\left(t-t_{0}\right)}}{\lambda^{2}} .
\end{aligned}
$$

Figure 8 shows the expectation and variance of $Z(t)$ for the same values of $\lambda, t_{0}, a$, and $b$ considered in Example 16 .

The computation of probabilities also can be performed directly through the 1-PDF. For instance, it may be of interest to compute the probability that the solution lies between two fixed values, say, $v_{1}=2$ and $v_{2}=3$ :

$$
\begin{aligned}
\mathbb{P}[2 \leq Z \leq 3] & =\int_{2}^{3} f_{1}(z, t) \mathrm{d} z \\
& = \begin{cases}-\mathrm{e}^{(\lambda / a)\left(b-(3 a+b) \mathrm{e}^{a\left(-t+t_{0}\right)}\right)}+\mathrm{e}^{(\lambda / a)\left(b-\mathrm{e}^{a\left(-t+t_{0}\right)}\left(b+a \operatorname{Max}\left[2, b\left(-1+\mathrm{e}^{a\left(t-t_{0}\right)}\right) / a\right]\right)\right)} & \text { if } \frac{b}{a}\left(\mathrm{e}^{a\left(t-t_{0}\right)}-1\right)<3, \\
0 & \text { if } \frac{b}{a}\left(\mathrm{e}^{a\left(t-t_{0}\right)}-1\right) \geq 3 .\end{cases}
\end{aligned}
$$




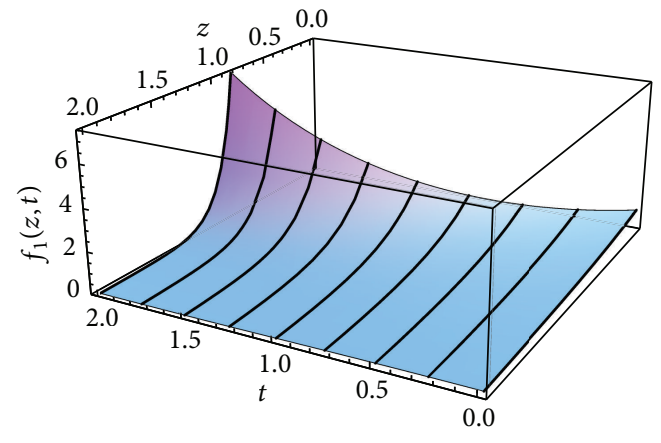

Figure 7: Plot of $f_{1}(z, t)$ given by (86) in Example 16 at different values of $t=\{0,0.25,0.5, \ldots, 2\}$ in the case that $Z_{0} \sim \operatorname{Exp}(1), t_{0}=0$, $a=-1$, and $b=1$.

5.2. Case III.2: B Is a Random Variable. Now we assume that only $B$ is a RV in the IVP $(1)$ and $f_{B}(b)$ denotes its PDF. For convenience, in this case, we rewrite (81) as follows:

$$
Z(t)=\frac{1}{a}\left(\mathrm{e}^{a\left(t-t_{0}\right)}-1\right) B+z_{0} \mathrm{e}^{a\left(t-t_{0}\right)}, \quad t \geq t_{0} \geq 0 .
$$

First, we fixed $t>t_{0}$ and denote $Z=Z(t)$. Then we again apply Proposition 2 to determine the 1-PDF $f_{1}(z, t)$ of $Z(t)$, taking into account that

$$
\begin{gathered}
\alpha=\frac{1}{a}\left(\mathrm{e}^{a\left(t-t_{0}\right)}-1\right)>0, \quad \beta=z_{0} \mathrm{e}^{a\left(t-t_{0}\right)}, \\
X=B, \quad Y=Z .
\end{gathered}
$$

Notice that $\alpha>0$ independently of the sign of the deterministic parameter $a \neq 0$. Then,

$$
\begin{array}{r}
f_{1}(z, t)=\frac{a}{\mathrm{e}^{a\left(t-t_{0}\right)}-1} f_{B}\left(\frac{a\left(z-z_{0} \mathrm{e}^{a\left(t-t_{0}\right)}\right)}{\mathrm{e}^{a\left(t-t_{0}\right)}-1}\right), \\
z_{1} \leq z \leq z_{2}, t>t_{0},
\end{array}
$$

where

$$
\begin{aligned}
& z_{1}=\mathrm{e}^{a\left(t-t_{0}\right)}\left(\frac{b_{1}}{a}+z_{0}\right)-\frac{b_{1}}{a}, \\
& z_{2}=\mathrm{e}^{a\left(t-t_{0}\right)}\left(\frac{b_{2}}{a}+z_{0}\right)-\frac{b_{2}}{a} .
\end{aligned}
$$

For $t=t_{0}$, as it also occurred in Cases I.2 and II.2, $Z(t)=$ $Z\left(t_{0}\right)=z_{0}$ and therefore

$$
f_{1}\left(z, t_{0}\right)=\delta\left(z-z_{0}\right), \quad-\infty<z<\infty .
$$

Example 18. Let us assume that $B$ follows a gamma distribution of parameters $\alpha, \beta>0$; that is, $B \sim \mathrm{Ga}(\alpha ; \beta)$. Therefore, according to (92)-(93), the 1-PDF of $Z(t)$ is given by

$$
\begin{aligned}
f_{1}(z, t)= & \frac{a}{\left(\mathrm{e}^{a\left(t-t_{0}\right)}-1\right) \beta^{\alpha} \Gamma(\alpha)}\left(\frac{a\left(z-z_{0} \mathrm{e}^{a\left(t-t_{0}\right)}\right)}{\mathrm{e}^{a\left(t-t_{0}\right)}-1}\right)^{\alpha-1} \\
& \times \mathrm{e}^{-a\left(z-z_{0}\right) \mathrm{e}^{a\left(t-t_{0}\right)} / \beta\left(\mathrm{e}^{a\left(t-t_{0}\right)}-1\right)} \\
& z_{0} \mathrm{e}^{a\left(t-t_{0}\right)}<z<+\infty, t>t_{0} .
\end{aligned}
$$

For each $t>t_{0}$, the domain of $z$ has been determined taking into account in (93) that in this case $b_{1}=0$ and $b_{2}=\infty$. It can be checked that $f_{1}(z, t)$ is a PDF for each $t>t_{0}$. Figure 9 shows $f_{1}(z, t)$ at different values of $t$ in the particular case that $B \sim \mathrm{Ga}(2 ; 4), t_{0}=0, z_{0}=1$, and $a=-1$. For $t=0$, according to (94), $f_{1}(z, 0)=\delta(z-1),-\infty<z<\infty$.

5.3. Case III.3: A Is a Random Variable. So far we have observed that the application of RVT method to determine the PDF of a RV, say $Y$, generated by a transformation $Y=r(X)$, relies strongly on the feasibility of computing the inverse of the map $y=r(x)$. Fortunately, this has been done exactly in the previous cases, but, in general, it is not possible in the current case where just $A$ is assumed to be a RV. In fact, once $t: t>t_{0}$ has been fixed, we must isolate $A$ in the equation:

$$
Z=r(A), \quad \text { where } r(A)=z_{0} \mathrm{e}^{A\left(t-t_{0}\right)}+\frac{b}{A}\left(\mathrm{e}^{A\left(t-t_{0}\right)}-1\right)
$$

which is not possible to perform in an exact manner. To circumvent this drawback, we will apply the LagrangeBürmann formula which gives the Taylor series expansion of the inverse of an analytic function.

Theorem 19 (Lagrange-Bürmann formula, see [10]). Suppose that $z$ is defined as a function of the variable $a$ by an equation of the form: $z=r(a)$ where $r$ is analytic about the point $a_{0}$ and $r^{\prime}\left(a_{0}\right) \neq 0$. Then, it is possible to invert (or to solve) the equation for $a: a=s(z)$ on a neighbourhood $\mathcal{N}\left(r\left(a_{0}\right) ; \delta\right), \delta>0$ of $r\left(a_{0}\right)$ :

$$
\begin{gathered}
a=s(z)=a_{0}+\sum_{n=1}^{\infty}\left(\lim _{a \rightarrow a_{0}}\left(\frac{d^{n-1}}{d a^{n-1}}\left(\frac{a-a_{0}}{r(a)-r\left(a_{0}\right)}\right)^{n}\right)\right. \\
\left.\times \frac{\left(z-r\left(a_{0}\right)\right)^{n}}{n !}\right), \\
z \in \mathcal{N}\left(r\left(a_{0}\right) ; \delta\right), \quad \delta>0 .
\end{gathered}
$$

Although this result permits obtaining, from a theoretical stand point, the inverse function of $r(a)$, in practice, often this can only be achieved in an approximate manner since the infinite series (97) must be truncated to be kept computationally feasible. Moreover, the representation (97) of the inverse is only valid in a certain neighbourhood $\mathcal{N}\left(r\left(a_{0}\right) ; \delta\right), \delta>0$ of $r\left(a_{0}\right)$, whose diameter $\delta>0$ must be determined carefully in each case study.

Let us apply Theorem 19 to determine the 1-PDF $f_{1}(z, t)$ of $Z(t)$. Fixing $t: t>t_{0}$ and denoting $Z=Z(t)$, we consider the map (96) which is analytic about any numerical value $0 \neq a=A(\omega), \omega \in \Omega$ on the assumed domain to the RV $A$. As the map (96), in general, is not monotone,we 


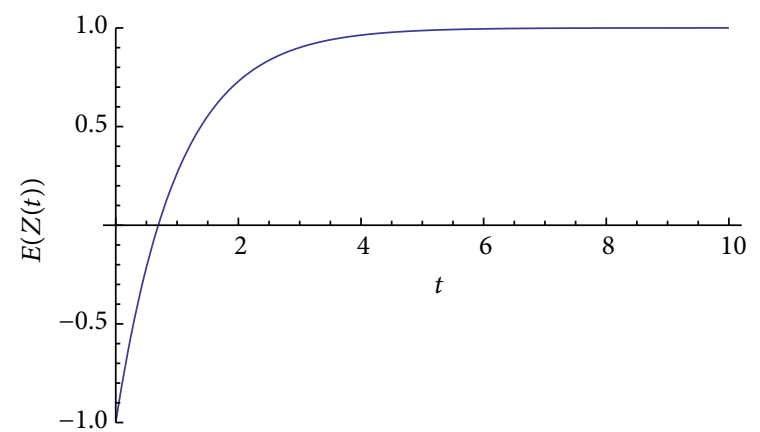

(a)

Figure 8: Expectation (a) and variance (b) of $Z(t)$ given by (88) in Example 17 in the case that $\lambda=1, t_{0}=0, a=-1$, and $b=1$.

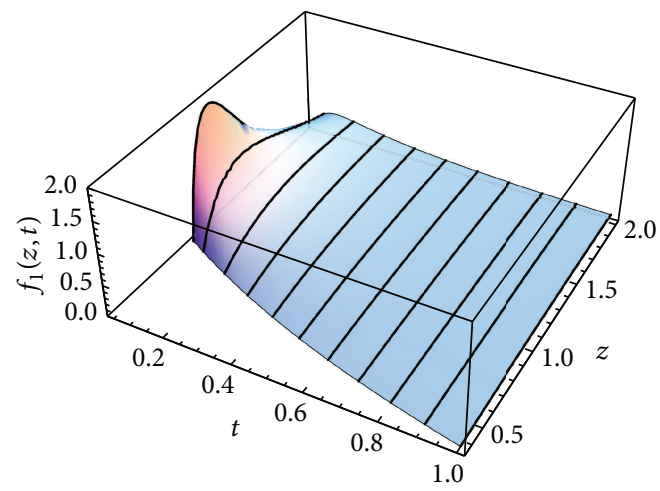

Figure 9: Plot of $f_{1}(z, t)$ given by (95) in Example 18 at different values of $t=\{0.05,0.1,0.2,0.3, \ldots, 1\}$ in the case that $B \sim$ $\mathrm{Ga}(2 ; 4), t_{0}=0, z_{0}=1$, and $a=-1$.

have to apply Theorem 1. In a first step, we divide the domain of the map $r$ (or equivalently, the domain of the RV $A$ ) into $k$ subintervals: $\mathscr{A}_{1}, \mathscr{A}_{2}, \ldots, \mathscr{A}_{k}$ where $r$ is monotone. Then, we will fix a such subinterval $\left.\mathscr{A}_{j}=\right] a_{\text {ini, }}, a_{\text {end, } j}[, 1 \leq$ $j \leq k$ where the contribution to the total 1-PDF $f_{1}(z, t)$ is going to be calculated. For this, we select a point $a_{0, j} \in \mathscr{A}_{j}$ where condition $r^{\prime}\left(a_{0, j}\right) \neq 0$ is met. By applying the LagrangeBürmann formula, we construct the inverse of the map $r(a)=$ $r_{j}(a)$ on $\mathscr{A}_{j}$ that, in the sequel, will be denoted by $s_{j}(z)$ :

$$
\begin{gathered}
s_{j}(z)=a_{0, j}+\sum_{n=1}^{\infty}\left(\lim _{a \rightarrow a_{0, j}}\left(\frac{\mathrm{d}^{n-1}}{\mathrm{~d} a^{n-1}}\left(\frac{a-a_{0, j}}{r(a)-r\left(a_{0, j}\right)}\right)^{n}\right)\right. \\
\left.\times \frac{\left(z-r\left(a_{0, j}\right)\right)^{n}}{n !}\right), \\
z \in \mathcal{N}\left(r\left(a_{0, j}\right) ; \delta\right), \delta>0 .
\end{gathered}
$$

In practice, this infinite series could only converge on a subset of $] r_{j}\left(a_{\mathrm{ini}, j}\right), r_{j}\left(a_{\mathrm{end}, j}\right)$ [ (if $r_{j}$ increases on $\left.\mathscr{A}_{j}\right)$ or ]$r_{j}\left(a_{\text {end }, j}\right), r_{j}\left(a_{\text {ini, } j}\right)$ ) (if $r_{j}$ decreases on $\left.\mathscr{A}_{j}\right)$. In such case,

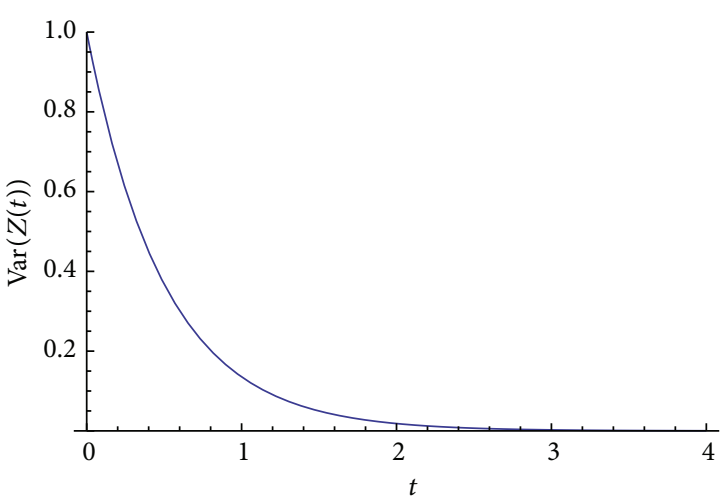

(b) the function $s_{j}(z)$ can be completed on the whole interval by taking $m_{j}$ another (or other, if necessary) appropriate point(s), say $a_{0_{1}, j} \in \mathscr{A}_{j}, 1 \leq l \leq m_{j}$ and then repeating the above process. In this manner, a piecewise inverse function $s_{j}(z)$ on $\mathscr{A}_{j}$ will be constructed.

In accordance with RVT method, besides constructing the inverse $s_{j}(z)$, one requires to compute its derivative. We again take advantage of Lagrange-Bürmann formula to complete this computation. In fact, notice that once $s_{j}(z)$ has been constructed, from (98), one gets

$$
\begin{gathered}
\frac{\mathrm{d} s_{j}(z)}{\mathrm{d} z}=\sum_{n=1}^{\infty}\left(\lim _{a \rightarrow a_{0, j}}\left(\frac{\mathrm{d}^{n-1}}{\mathrm{~d} a^{n-1}}\left(\frac{a-a_{0, j}}{r(a)-r\left(a_{0, j}\right)}\right)^{n}\right)\right. \\
\left.\times \frac{\left(z-r\left(a_{0, j}\right)\right)^{n-1}}{(n-1) !}\right), \\
z \in \mathcal{N}\left(r\left(a_{0, j}\right) ; \delta\right), \quad \delta>0 .
\end{gathered}
$$

Notice that if $s_{j}(z)$ has been defined by means of a piecewise function, then $\mathrm{d} s_{j}(z) / \mathrm{d} z$ will also be defined in the same way.

The process will be culminated by repeating again the previous argument on all the subintervals $\mathscr{A}_{1}, \mathscr{A}_{2}, \ldots, \mathscr{A}_{k}$. Following the previous development, the 1-PDF $f_{1}(z, t)$ of $Z(t)$ can be computed as follows:

$$
f_{1}(z, t)=\sum_{j=1}^{k} f_{A}\left(s_{j}(z)\right)\left|\frac{\mathrm{d} s_{j}(z)}{\mathrm{d} z}\right|,
$$

where $s_{j}(z)$ and $\mathrm{d} s_{j}(z) / \mathrm{d} z$ are defined by (98) and (99), respectively. 
Often, the infinite series (98) has to be truncated at the term $N_{j}$ to control computational burden. In this way, we obtain an approximation of the inverse:

$$
\begin{gathered}
s_{j, N_{j}}(z) \\
=a_{0, j}+\sum_{n=1}^{N_{j}}\left(\lim _{a \rightarrow a_{0, j}}\left(\frac{\mathrm{d}^{n-1}}{\mathrm{~d} a^{n-1}}\left(\frac{a-a_{0, j}}{r(a)-r\left(a_{0, j}\right)}\right)^{n}\right)\right. \\
\left.\times \frac{\left(z-r\left(a_{0, j}\right)\right)^{n}}{n !}\right) .
\end{gathered}
$$

Thus, an approximation of its derivative is

$$
\begin{aligned}
& \frac{\mathrm{d} s_{j, N_{j}}(z)}{\mathrm{d} z} \\
& =\sum_{n=1}^{N_{j}}\left(\lim _{a \rightarrow a_{0, j}}\left(\frac{\mathrm{d}^{n-1}}{\mathrm{~d} a^{n-1}}\left(\frac{a-a_{0, j}}{r(a)-r\left(a_{0, j}\right)}\right)^{n}\right)\right. \\
& \left.\quad \times \frac{\left(z-r\left(a_{0, j}\right)\right)^{n-1}}{(n-1) !}\right) .
\end{aligned}
$$

The infinite series (98) and (99) have the same convergence radius; however, often in practice, we need to handle their corresponding truncations (101) and (102), respectively. In this case, the selection of the appropriate $a_{0 l}, j$ depends also on the quality of the approximations provided by both truncated series.

Repeating the foregoing process on each interval $\mathscr{A}_{j}, 1 \leq$ $j \leq k$, one gets the corresponding approximation of $f_{1}(z, t)$ given by

$$
f_{1}(z, t)=\sum_{j=1}^{k} f_{A}\left(s_{j, N_{j}}(z)\right)\left|\frac{\mathrm{d} s_{j, N_{j}}(z)}{\mathrm{d} z}\right| .
$$

In the following example, we illustrate the previous development.

Example 20. Let us assume that $A$ has a beta distribution of parameters $\alpha=2, \beta=3, A \sim \operatorname{Be}(2 ; 3), t_{0}=0$, $z_{0}=1$ and $b=1$. Figure 10 shows the approximation of $f_{1}(z, t)$ at different values of $t$. The approximation has been performed by (103), (101), and (102) with $k=1$ being $\mathscr{A}_{1}=$ $[0,1]$ because of monotony of $r(A)$. In order to carry out the computations, $\mathscr{A}_{1}$ has been split into 7 subintervals in accordance with the process described previously. In each subinterval, an approximation of degree $N_{j}=2$ has been used.

5.4. Case III.4: $\left(Z_{0}, B\right)$ Is a Random Vector. Let us consider the IVP (1) and suppose that both $Z_{0}$ and $B$ are continuous RVs with joint PDF $f_{Z_{0}, B}\left(z_{0}, b\right)$. We rewrite $(81)$ in the equivalent form:

$$
\begin{aligned}
& Z(t)=Z_{1}(t)+Z_{2}(t), \\
& \text { where }\left\{\begin{array}{l}
Z_{1}(t)=Z_{0} \mathrm{e}^{a\left(t-t_{0}\right)} \\
Z_{2}(t)=B\left(\frac{1}{a}\left(\mathrm{e}^{a\left(t-t_{0}\right)}-1\right)\right) .
\end{array}\right.
\end{aligned}
$$

In order to compute the 1-PDF of $Z(t), f_{1}(z, t)$, we first fix $t: t>t_{0}$ and consider the continuous RVs $Z_{1}=Z_{1}(t)$, $Z_{2}=Z_{2}(t)$, and $Z=Z_{1}+Z_{2}=Z(t)$. Then, we will apply Propositions 6 and 7. Indeed, in a first step, we compute the joint PDF $f_{Z_{1}, Z_{2}}\left(z_{1}, z_{2}\right)$ by applying Proposition 6 to

$$
\begin{gathered}
X_{1}=Z_{0}, \quad X_{2}=B, \quad r_{1}\left(z_{0}\right)=z_{0} \mathrm{e}^{a\left(t-t_{0}\right)}, \\
r_{2}(b)=b\left(\frac{1}{a}\left(\mathrm{e}^{a\left(t-t_{0}\right)}-1\right)\right), \quad Y_{1}=Z_{1}, \\
Y_{2}=Z_{2}, \quad s_{1}\left(z_{1}\right)=z_{1} \mathrm{e}^{-a\left(t-t_{0}\right)}, \\
s_{2}\left(z_{2}\right)=z_{2} \frac{a}{\mathrm{e}^{a\left(t-t_{0}\right)}-1}
\end{gathered}
$$

and taking into account that

$$
\frac{\mathrm{d} s_{1}\left(z_{1}\right)}{\mathrm{d} z_{1}}=\mathrm{e}^{-a\left(t-t_{0}\right)}>0, \quad \frac{\mathrm{d} s_{2}\left(z_{2}\right)}{\mathrm{d} z_{2}}=\frac{a}{\mathrm{e}^{a\left(t-t_{0}\right)}-1}>0 .
$$

This leads to

$$
\begin{gathered}
f_{Z_{1}, Z_{2}}\left(z_{1}, z_{2}\right)=f_{Z_{0}, B}\left(z_{1} \mathrm{e}^{-a\left(t-t_{0}\right)}, \frac{z_{2} a}{\mathrm{e}^{a\left(t-t_{0}\right)}-1}\right) \frac{a \mathrm{e}^{-a\left(t-t_{0}\right)}}{\mathrm{e}^{a\left(t-t_{0}\right)}-1}, \\
z_{1,1} \leq z_{1} \leq z_{1,2}, z_{2,1} \leq z_{2} \leq z_{2,2},
\end{gathered}
$$

where

$$
\begin{aligned}
& z_{1,1}=z_{0,1} \mathrm{e}^{a\left(t-t_{0}\right)}, \quad z_{1,2}=z_{0,2} \mathrm{e}^{a\left(t-t_{0}\right)}, \\
& z_{2,1}=\frac{b_{1}}{a}\left(\mathrm{e}^{a\left(t-t_{0}\right)}-1\right), \quad z_{2,2}=\frac{b_{2}}{a}\left(\mathrm{e}^{a\left(t-t_{0}\right)}-1\right) .
\end{aligned}
$$

Notice that to determine the variation of $z_{2}$, we have used that $1 / a\left(e^{a\left(t-t_{0}\right)}-1\right)>0$ for each $a$ and $t: t>t_{0}$. Finally, we apply Proposition 7 using the following identification: $X_{1}=$ $Z_{1}, X_{2}=Z_{2}$, and $Y_{1}=Z$. This yields

$$
f_{1}(z, t)=\int_{z_{1,1}}^{z_{1,2}} f_{Z_{1}, Z_{2}}\left(z_{1}, z-z_{1}\right) \mathrm{d} z_{1},
$$

or more explicitly, using (107),

$$
\begin{aligned}
& f_{1}(z, t) \\
& =\int_{z_{1,1}}^{z_{1,2}} f_{Z_{0}, B}\left(z_{1} \mathrm{e}^{-a\left(t-t_{0}\right)}, \frac{a\left(z-z_{1}\right)}{\mathrm{e}^{a\left(t-t_{0}\right)}-1}\right) \frac{a \mathrm{e}^{-a\left(t-t_{0}\right)}}{\mathrm{e}^{a\left(t-t_{0}\right)}-1} \mathrm{~d} z_{1}, \\
& z_{1,1}+z_{2,1} \leq z \leq z_{1,2}+z_{2,2}, t>t_{0},
\end{aligned}
$$

where $z_{1,1}, z_{1,2}, z_{2,1}$, and $z_{2,2}$ are defined by (108). 


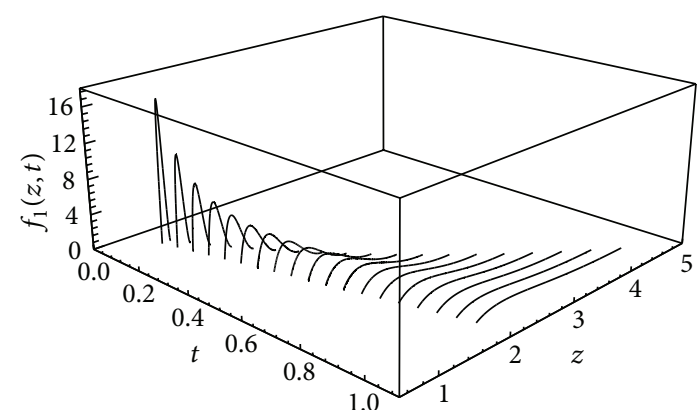

(a)

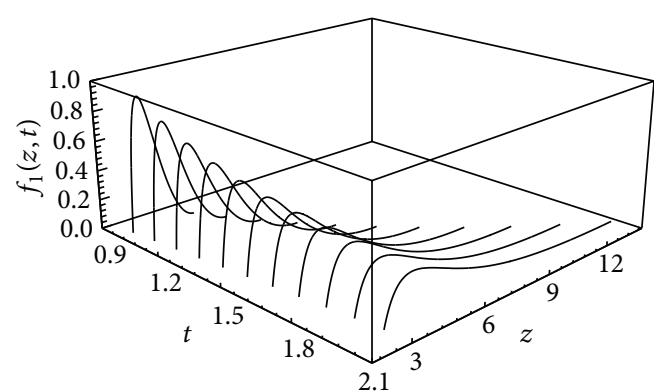

(b)

Figure 10: Plot of $f_{1}(z, t)$ given by (96) in Example 20 at different values of $t=\{0,0.1,0.2, \ldots, 1\}$ (a) and $t=\{0.9,1,1.1, \ldots, 2\}$ (b) in the case that $A \sim \operatorname{Be}(2 ; 3), t_{0}=0, z_{0}=1$, and $b=1$. In order to highlight better the shape of $f_{1}(z, t)$, we have split its representation into two plots. Notice the different scales in the vertical axes for each plot.

If $t=t_{0}$, then $Z(t)=Z\left(t_{0}\right)=Z_{0}$ and, similar to Cases I.3 and II. $3, f_{1}\left(z_{0}, t_{0}\right)$ is the PDF of RV $Z_{0}$. It is obtained as the following marginal distribution of the $f_{Z_{0}, B}\left(z_{0}, b\right)$ :

$$
\begin{array}{r}
f_{1}\left(z_{0}, t_{0}\right)=f_{Z_{0}}\left(z_{0}\right)=\int_{b_{1}}^{b_{2}} f_{Z_{0}, B}\left(z_{0}, b\right) \mathrm{d} b, \\
z_{0,1} \leq z_{0} \leq z_{0,2} .
\end{array}
$$

Example 21. Let $\left(Z_{0}, B\right)$ be a two-dimensional Gaussian vector, $\eta=\left(Z_{0}, B\right)^{T} \sim N\left(\mu_{\eta}, \Sigma_{\eta}\right)$, where

$$
\mu_{\eta}=\left(\begin{array}{c}
\mu_{Z_{0}} \\
\mu_{B}
\end{array}\right), \quad \Sigma_{\eta}=\left(\begin{array}{cc}
\left(\sigma_{Z_{0}}\right)^{2} & \rho_{Z_{0}, B} \sigma_{Z_{0}} \sigma_{B} \\
\rho_{Z_{0}, B} \sigma_{Z_{0}} \sigma_{B} & \left(\sigma_{B}\right)^{2}
\end{array}\right),
$$

and $\rho_{Z_{0}, B}$ denotes the correlation coefficient between $Z_{0}$ and $B$. Then, according to (110) and (108), the 1-PDF of $Z(t)$ is given by

$$
\begin{aligned}
f_{1}(z, t)= & \frac{1}{2 \pi \sqrt{\operatorname{det}\left(\Sigma_{\eta}\right)}} \\
& \times \int_{-\infty}^{+\infty} \mathrm{e}^{-(1 / 2)\left(\zeta-\mu_{\eta}\right)^{T}\left(\Sigma_{\eta}\right)^{-1}\left(\zeta-\mu_{\eta}\right)} \frac{a \mathrm{e}^{-a\left(t-t_{0}\right)}}{\mathrm{e}^{a\left(t-t_{0}\right)}-1} \mathrm{~d} z_{1},
\end{aligned}
$$

where

$$
\zeta=\left(\begin{array}{c}
z_{1} \mathrm{e}^{-a\left(t-t_{0}\right)} \\
\frac{a\left(z-z_{1}\right)}{\mathrm{e}^{a\left(t-t_{0}\right)}-1}
\end{array}\right)
$$

Notice that to determine the integration limits in (113), we have considered in (108) that $z_{0,1}=-\infty$ and $z_{0,2}=+\infty$. Figure 11 shows $f_{1}(z, t)$ at different values of $t$ in the case that

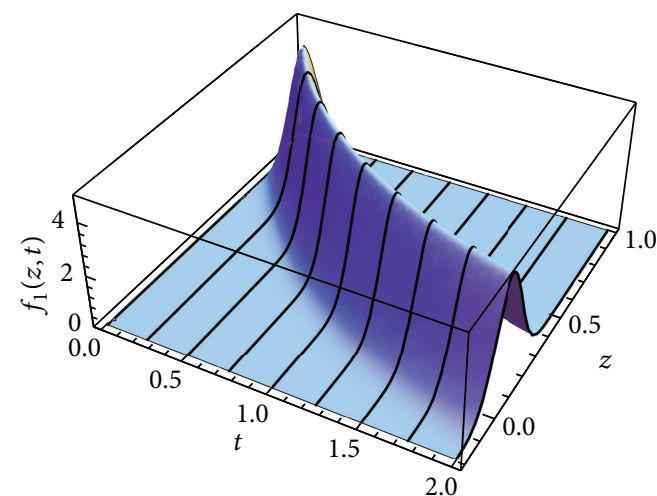

FigURE 11: Plot of $f_{1}(z, t)$ given by (113)-(114) in Example 21 at different values of $t=\{0,0.25,0.5, \ldots, 2\}$ in the case that $\eta=$ $\left(Z_{0}, B\right)^{T} \sim N\left(\mu_{\eta}, \Sigma_{\eta}\right)$ where $\mu_{\eta}$ and $\Sigma_{\eta}$ are given by (112) with $\mu_{Z_{0}}=1$, $\mu_{B}=0, \sigma_{Z_{0}}=0.1, \sigma_{B}=0.1, \rho_{Z_{0} B}=0.5, t_{0}=0$, and $a=-1$.

$\mu_{Z_{0}}=1, \mu_{B}=0, \sigma_{Z_{0}}=0.1, \sigma_{B}=0.1, \rho_{Z_{0}, B}=0.5, t_{0}=0$, and $a=-1$.

For $t=0$, as the marginal distribution of a bivariate Gaussian distribution is also a Gaussian distribution with mean and variance of the corresponding component of the random vector, one gets

$$
\begin{array}{r}
f_{1}\left(z_{0}, 0\right)=f_{Z_{0}}\left(z_{0}\right)=\frac{1}{\sqrt{2 \pi\left(\sigma_{z_{0}}\right)^{2}}} \mathrm{e}^{-(1 / 2)\left(\left(z_{0}-\mu_{z_{0}}\right) / \sigma_{z_{0}}\right)^{2}}, \\
-\infty<z_{0}<\infty .
\end{array}
$$

5.5. Case III.5: $\left(Z_{0}, A\right)$ Is a Random Vector. Let us consider the IVP (1) and now we assume that both $Z_{0}$ and $A$ are 
continuous RVs with joint $\operatorname{PDF} f_{Z_{0}, A}\left(z_{0}, a\right)$. We rewrite (81) in the following equivalent form:

$$
\begin{aligned}
& Z(t)=Z_{1}(t)+Z_{2}(t), \\
& \text { where }\left\{\begin{array}{l}
Z_{1}(t)=\left(Z_{0}+\frac{b}{A}\right) \mathrm{e}^{A\left(t-t_{0}\right)}, \\
Z_{2}(t)=-\frac{b}{A} .
\end{array}\right.
\end{aligned}
$$

In order to apply RVT, we fix $t: t \geq t_{0}$ and denote $Z_{1}=Z_{1}(t)$, $Z_{2}=Z_{2}(t)$, and $Z=Z_{1}+Z_{2}=Z(t)$. To compute the PDF of $Z$, first we will determine the joint PDF of $Z_{1}$ and $Z_{2}$ by applying Theorem 5 to

$$
\begin{aligned}
& X_{1}=Z_{0}, \quad X_{2}=A, \quad r_{1}\left(z_{0}, a\right)=\left(z_{0}+\frac{b}{a}\right) \mathrm{e}^{a\left(t-t_{0}\right)}, \\
& r_{2}(a)=-\frac{b}{a}, \quad Y_{1}=Z_{1}, \quad Y_{2}=Z_{2}, \\
& s_{1}\left(z_{1}, z_{2}\right)=z_{1} \mathrm{e}^{\left(b / z_{2}\right)\left(t-t_{0}\right)}+z_{2}, \quad s_{2}\left(z_{2}\right)=-\frac{b}{z_{2}} .
\end{aligned}
$$

Then taking into account $\partial s_{2}\left(z_{2}\right) / \partial z_{1}=0$, the involved Jacobian simplifies to

$$
\left|J_{2}\right|=\frac{|b|}{\left(z_{2}\right)^{2}} \mathrm{e}^{b\left(t-t_{0}\right) / z_{2}}>0
$$

Therefore,

$$
\begin{aligned}
f_{Z_{1}, Z_{2}}\left(z_{1}, z_{2}\right) & =\frac{|b|}{\left(z_{2}\right)^{2}} \mathrm{e}^{b\left(t-t_{0}\right) / z_{2}} f_{Z_{0}, A} \\
& \times\left(z_{1} \mathrm{e}^{\left(b / z_{2}\right)\left(t-t_{0}\right)}+z_{2},-\frac{b}{z_{2}}\right), \\
z_{1,1} \leq & z_{1} \leq z_{1,2}, \quad z_{2,1} \leq z_{2} \leq z_{2,2},
\end{aligned}
$$

where

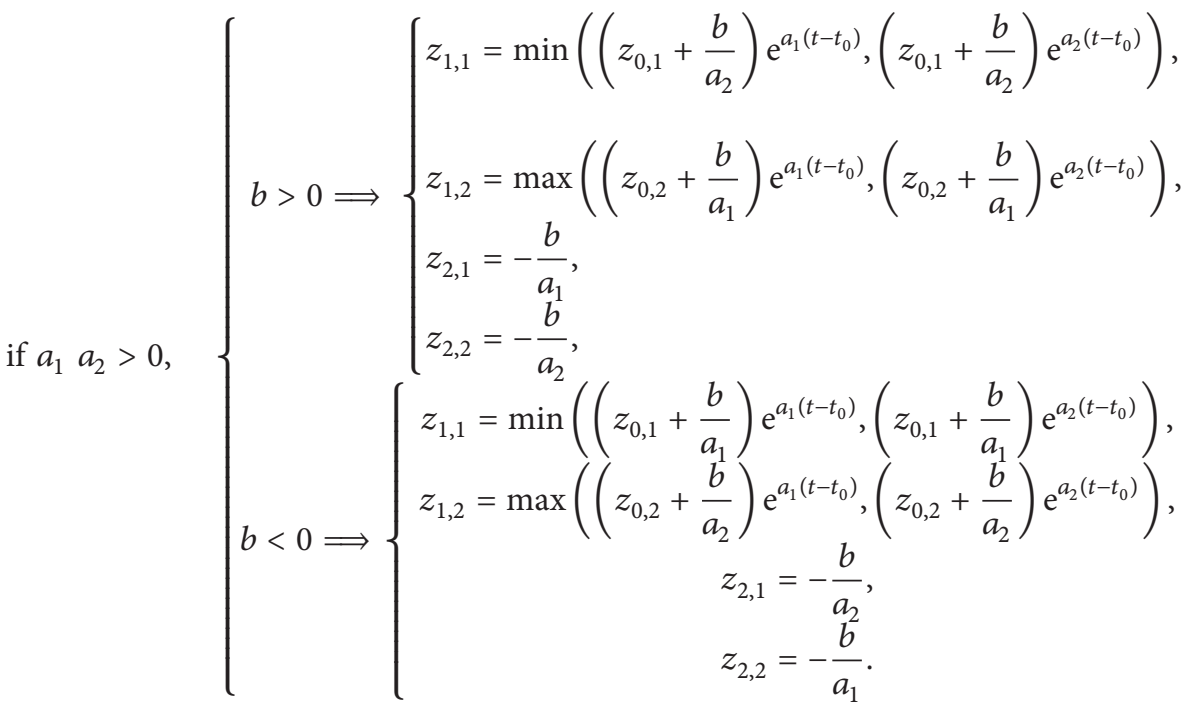

In the case that $a_{1} a_{2}<0$, the computation of the domain of variation of $z_{1}$ and $z_{2}$ in (119) is more complicated. To express it, we first introduce the numbers:

$$
\begin{aligned}
& a_{0^{-}}=\sup \{a=A(\omega): a<0, \omega \in \Omega\}, \\
& a_{0^{+}}=\inf \{a=A(\omega): a>0, \omega \in \Omega\} .
\end{aligned}
$$

Then, after elaborated computations, one gets

$$
\text { if } a_{1} a_{2}<0, \quad\left\{\begin{array}{l}
b>0 \Longrightarrow\left\{\begin{array}{l}
z_{1} \in I_{1,1}^{+} \cup I_{1,2}^{+}, \\
z_{2} \in I_{2,1}^{+} \cup I_{2,2}^{+},
\end{array}\right. \\
b<0 \Longrightarrow\left\{\begin{array}{l}
z_{1} \in I_{1,1}^{-} \cup I_{1,2}^{-}, \\
z_{2} \in I_{2,1}^{-} \cup I_{2,2}^{-}
\end{array}\right.
\end{array}\right.
$$

where

$$
\begin{aligned}
I_{1,1}^{+}= & {\left[\min \left(\left(z_{0,1}+\frac{b}{a_{0^{-}}}\right) \mathrm{e}^{a_{1}\left(t-t_{0}\right)},\left(z_{0,1}+\frac{b}{a_{0^{-}}}\right) \mathrm{e}^{a_{0^{-}}\left(t-t_{0}\right)}\right),\right.} \\
& \left.\max \left(\left(z_{0,2}+\frac{b}{a_{1}}\right) \mathrm{e}^{a_{1}\left(t-t_{0}\right)},\left(z_{0,2}+\frac{b}{a_{1}}\right) \mathrm{e}^{a_{0^{-}}\left(t-t_{0}\right)}\right)\right], \\
I_{1,2}^{+}= & {\left[\min \left(\left(z_{0,1}+\frac{b}{a_{2}}\right) \mathrm{e}^{a_{0^{+}}\left(t-t_{0}\right)},\left(z_{0,1}+\frac{b}{a_{2}}\right) \mathrm{e}^{a_{2}\left(t-t_{0}\right)}\right),\right.} \\
& \left.\max \left(\left(z_{0,2}+\frac{b}{a_{0^{+}}}\right) \mathrm{e}^{a_{0^{+}}\left(t-t_{0}\right)},\left(z_{0,2}+\frac{b}{a_{0^{+}}}\right) \mathrm{e}^{a_{2}\left(t-t_{0}\right)}\right)\right],
\end{aligned}
$$




$$
\begin{gathered}
I_{1,1}^{-}=\left[\min \left(\left(z_{0,1}+\frac{b}{a_{1}}\right) \mathrm{e}^{a_{1}\left(t-t_{0}\right)},\left(z_{0,1}+\frac{b}{a_{1}}\right) \mathrm{e}^{a_{0^{-}}\left(t-t_{0}\right)}\right),\right. \\
\left.\max \left(\left(z_{0,2}+\frac{b}{a_{0^{-}}}\right) \mathrm{e}^{a_{1}\left(t-t_{0}\right)},\left(z_{0,2}+\frac{b}{a_{0^{-}}}\right) \mathrm{e}^{a_{0}\left(t-t_{0}\right)}\right)\right], \\
I_{1,2}^{-}=\left[\min \left(\left(z_{0,1}+\frac{b}{a_{0^{+}}}\right) \mathrm{e}^{a_{0^{+}}\left(t-t_{0}\right)},\left(z_{0,1}+\frac{b}{a_{0^{+}}}\right) \mathrm{e}^{a_{2}\left(t-t_{0}\right)}\right),\right. \\
\left.\quad \max \left(\left(z_{0,2}+\frac{b}{a_{2}}\right) \mathrm{e}^{a_{0^{+}}\left(t-t_{0}\right)},\left(z_{0,2}+\frac{b}{a_{2}}\right) \mathrm{e}^{a_{2}\left(t-t_{0}\right)}\right)\right], \\
I_{2,1}^{+}=\left[-\frac{b}{a_{0^{+}}},-\frac{b}{a_{2}}\right], \quad I_{2,2}^{+}=\left[-\frac{b}{a_{1}},-\frac{b}{a_{0^{-}}}\right], \\
I_{2,1}^{-}=\left[-\frac{b}{a_{0^{-}}},-\frac{b}{a_{1}}\right], \quad I_{2,2}^{-}=\left[-\frac{b}{a_{2}},-\frac{b}{a_{0^{+}}}\right] .
\end{gathered}
$$

Notice that $a_{0^{-}}$and/or $a_{0^{+}}$can become 0 . In this case, the extremes of the intervals above that depend on $a_{0^{-}}$and/or $a_{0^{+}}$ become $\pm \infty$, depending on the sign of parameter $b$.

Once the joint PDF of $\left(Z_{1}, Z_{2}\right)$ has been determined, the computation of the PDF of $Z=Z_{1}+Z_{2}$ follows directly by applying Proposition 7. Indeed, as $Z_{2}=-b / A \neq 0$ (since into Case III, $b \neq 0$; otherwise we would be in Case I), we will apply formula (22) to $X_{1}=Z_{1}=\left(Z_{0}+(b / A)\right) \mathrm{e}^{A\left(t-t_{0}\right)}, X_{2}=Z_{2}=$ $-b / A \neq 0$, and $Y_{1}=Z=Z_{1}+Z_{2}$. This yields

$$
f_{1}(z, t)=f_{Z}(z)=\int_{D_{Z_{2}}} f_{Z_{1}, Z_{2}}\left(z-z_{2}, z_{2}\right) \mathrm{d} z_{2}
$$

where $D_{Z_{2}}$ denotes the domain of variation of $Z_{2}$ which, according to (120)-(124), depends on both the sign of the products $a_{1} a_{2}$ and $b$. Using (119), it is equivalent to

$$
\begin{gathered}
f_{1}(z, t) \\
=\int_{D_{Z_{2}}} \frac{|b|}{\left(z_{2}\right)^{2}} \mathrm{e}^{b\left(t-t_{0}\right) / z_{2}} \\
\times f_{Z_{0}, A}\left(z \mathrm{e}^{\left(b / z_{2}\right)\left(t-t_{0}\right)}+z_{2}\left(1-\mathrm{e}^{\left(b / z_{2}\right)\left(t-t_{0}\right)}\right),\right. \\
\left.-\frac{b}{z_{2}}\right) \mathrm{d} z_{2}, \\
z \in D_{Z}, t \geq t_{0} .
\end{gathered}
$$

The range of variation of $Z$, denoted by $D_{Z}$, can be straightforwardly computed taking into account $Z=Z_{1}+Z_{2}$ and the domains of $Z_{1}$ and $Z_{2}$ which have been determined previously in (120)-(124). As its practical determination in specific examples is simple from the previous exposition, in order to avoid an unwieldy notation, we do not rewrite the final general expression.
Example 22. Let $\left(Z_{0}, A\right)$ be a two-dimensional Gaussian vector, $\eta=\left(Z_{0}, A\right)^{T} \sim N\left(\mu_{\eta}, \Sigma_{\eta}\right)$, where

$$
\mu_{\eta}=\left(\begin{array}{c}
\mu_{Z_{0}} \\
\mu_{A}
\end{array}\right), \quad \Sigma_{\eta}=\left(\begin{array}{cc}
\left(\sigma_{Z_{0}}\right)^{2} & \rho_{Z_{0}, A} \sigma_{Z_{0}} \sigma_{A} \\
\rho_{Z_{0}, A} \sigma_{Z_{0}} \sigma_{A} & \left(\sigma_{A}\right)^{2}
\end{array}\right),
$$

And let $\rho_{Z_{0}, A}$ denotes the correlation coefficient between $Z_{0}$ and $A$. Then, according to (126), (122), and (124) to determine the domain of $z_{2}$, the 1-PDF of $Z(t)$ is given by

$$
\begin{aligned}
f_{1}(z, t)= & \frac{|b|}{2 \pi \sqrt{\operatorname{det}\left(\Sigma_{\eta}\right)}}, \\
& \times \int_{-\infty}^{+\infty} \frac{1}{\left(z_{2}\right)^{2}} \mathrm{e}^{b\left(t-t_{0}\right) / z_{2}} \mathrm{e}^{-(1 / 2)\left(\zeta-\mu_{\eta}\right)^{T}\left(\Sigma_{\eta}\right)^{-1}\left(\zeta-\mu_{\eta}\right)} \mathrm{d} z_{2},
\end{aligned}
$$

where

$$
\zeta=\left(\begin{array}{c}
z \mathrm{e}^{\left(b / z_{2}\right)\left(t-t_{0}\right)}+z_{2}\left(1-\mathrm{e}^{\left(b / z_{2}\right)\left(t-t_{0}\right)}\right) \\
-\frac{b}{z_{2}}
\end{array}\right) .
$$

Hereinafter, we will assume that $b>0$ to facilitate our discussion through the example. We are in the case that $z_{0,1}=$ $-\infty, z_{0,2}=+\infty$, and $a_{1}=-\infty ; a_{2}=+\infty$; hence, $a_{1} a_{2}<0$, $a_{0^{-}}=0^{-}$, and $a_{0^{+}}=0^{+}$; thus, $z_{2,1}=-\infty$ and $z_{2,2}=+\infty$, which, according to (126), (122), and (124), determines the limits of integration of (128). Specifically,

$$
\begin{aligned}
Z_{2} \in I_{2,1}^{+} \cup I_{2,2}^{+} & =\left[-\frac{b}{a_{0^{+}}},-\frac{b}{a_{2}}\right] \cup\left[-\frac{b}{a_{1}},-\frac{b}{a_{0^{-}}}\right] \\
& =\left[-\frac{b}{0^{+}},-\frac{b}{+\infty}\right] \cup\left[-\frac{b}{-\infty},-\frac{b}{0^{-}}\right] \\
& =]-\infty, 0] \cup[0,+\infty[=]-\infty,+\infty[.
\end{aligned}
$$

Following an analogous reasoning, it is easy to check from (121) and (123) that $\left.I_{1,1}^{+}=\right]-\infty,+\infty\left[\right.$ and $\left.I_{1,2}^{+}=\right]-\infty,+\infty[$. Therefore, the domain of variation to $Z_{1}$ is ] $-\infty,+\infty$ [. As a consequence, in (128), $Z$ lies in $-\infty \leq z \leq+\infty$.

Figure 12 shows $f_{1}(z, t)$ at different values of $t$ in the case that $\mu_{Z_{0}}=1, \mu_{A}=0, \sigma_{Z_{0}}=0.1, \sigma_{A}=0.1, \rho_{Z_{0}, A}=0.5, t_{0}=0$, and $b=1$.

5.6. Case III.6: $(B, A)$ Is a Random Vector. Let us consider the IVP (1) and let us assume that $B$ and $A$ are continuous RVs with joint $\mathrm{PDF} f_{B, A}(b, a)$. For convenience, we rewrite (81) in the following equivalent form:

$$
\begin{aligned}
& Z(t)=Z_{1}(t)+Z_{2}(t), \\
& \text { where }\left\{\begin{array}{l}
Z_{1}(t)=\frac{B}{A}\left(\mathrm{e}^{A\left(t-t_{0}\right)}-1\right), \\
Z_{2}(t)=z_{0} \mathrm{e}^{A\left(t-t_{0}\right)} .
\end{array}\right.
\end{aligned}
$$




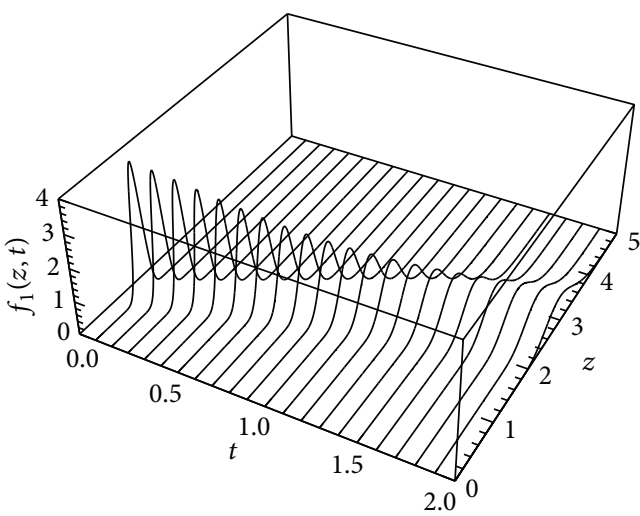

(a)

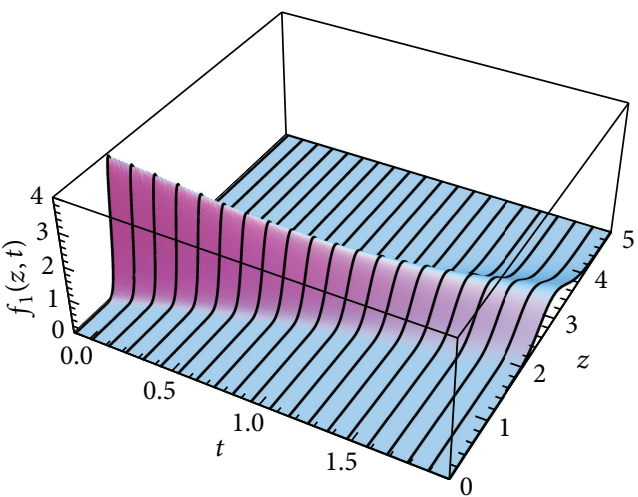

(b)

Figure 12: Plot of $f_{1}(z, t)$ given by (128)-(129) in Example 22 at different values of $t=\{0,0.1,0.2, \ldots, 2\}$ in the case that $\eta=\left(Z_{0}, A\right)^{T} \sim$ $N\left(\mu_{\eta}, \Sigma_{\eta}\right)$ where $\mu_{\eta}$ and $\Sigma_{\eta}$ are given by (127) with $\mu_{Z_{0}}=1, \mu_{A}=0, \sigma_{Z_{0}}=0.1, \sigma_{A}=0.1, \rho_{Z_{0}, A}=0.5, t_{0}=0$, and $b=1$.

In order to apply RVT method, we fix $t: t>t_{0}$ and denote $Z_{1}=Z_{1}(t), Z_{2}=Z_{2}(t)$, and $Z=Z_{1}+Z_{2}=Z(t)$. We first consider the case where $z_{0} \neq 0$. To compute the $\operatorname{PDF}$ of $Z$, first we will determine the joint PDF of $Z_{1}$ and $Z_{2}$ by applying Theorem 5 to

$$
\begin{gathered}
X_{1}=B, \quad X_{2}=A, \quad r_{1}(b, a)=\frac{b}{a}\left(\mathrm{e}^{a\left(t-t_{0}\right)}-1\right), \\
r_{2}(a)=z_{0} \mathrm{e}^{a\left(t-t_{0}\right)}, \quad Y_{1}=Z_{1}, \quad Y_{2}=Z_{2}, \\
s_{1}\left(z_{1}, z_{2}\right)=\frac{z_{0} z_{1}}{t-t_{0}} \frac{\ln \left|z_{2}\right|-\ln \left|z_{0}\right|}{z_{2}-z_{0}}, \\
s_{2}\left(z_{2}\right)=\frac{\ln \left|z_{2}\right|-\ln \left|z_{0}\right|}{t-t_{0}} .
\end{gathered}
$$

As $\partial a / \partial z_{1}=0$, in order to compute the Jacobian $J_{2}$, it is enough to calculate the two following partial derivatives:

$$
\frac{\partial b}{\partial z_{1}}=\frac{z_{0}}{t-t_{0}} \frac{\ln \left|z_{2}\right|-\ln \left|z_{0}\right|}{z_{2}-z_{0}} \neq 0, \quad \frac{\partial a}{\partial z_{2}}=\frac{1}{z_{2}\left(t-t_{0}\right)} \neq 0,
$$

which are well defined since $t>t_{0}, z_{2}=z_{0} \mathrm{e}^{a\left(t-t_{0}\right)} \neq 0$, and $z_{2}-$ $z_{0}=z_{0}\left(\mathrm{e}^{a\left(t-t_{0}\right)}-1\right) \neq 0$ due to by hypothesis $z_{0} \neq 0$ and $\mathbb{P}[\{\omega \in$ $\Omega: A(\omega) \neq 0\}]=1$. Also notice that $\ln \left|z_{2}\right|-\ln \left|z_{0}\right|=a(t-$ $\left.t_{0}\right) \neq 0$. Moreover, taking into account that $z_{0} / z_{2}=\mathrm{e}^{-a\left(t-t_{0}\right)}>$ 0 , one gets

$$
\begin{aligned}
f_{Z_{1}, Z_{2}} & \left(z_{1}, z_{2}\right) \\
= & f_{B, A}\left(\frac{z_{0} z_{1}}{t-t_{0}} \frac{\ln \left|z_{2}\right|-\ln \left|z_{0}\right|}{z_{2}-z_{0}}, \frac{\ln \left|z_{2}\right|-\ln \left|z_{0}\right|}{t-t_{0}}\right) \\
& \times \frac{1}{\left(t-t_{0}\right)^{2}} \frac{z_{0}}{z_{2}}\left|\frac{\ln \left|z_{2}\right|-\ln \left|z_{0}\right|}{z_{2}-z_{0}}\right|, \\
& z_{1,1} \leq z_{1} \leq z_{1,2}, \quad z_{2,1} \leq z_{2} \leq z_{2,2} .
\end{aligned}
$$

The values $z_{1,1}, z_{1,2}, z_{2,1}$, and $z_{2,2}$ that determine the domain of variation of $Z_{1}$ and $Z_{2}$ can be computed taking into account that the function $h_{1}(a)=(1 / a)\left(\mathrm{e}^{a\left(t-t_{0}\right)}-1\right)$ is positive and increasing on the whole real line in the current case where $t-t_{0}>0$. This yields

$$
\text { if } a_{1} a_{2}>0 \text {, and }\left\{\begin{aligned}
& b_{1}>0 \text { or } b_{1} b_{2}<0 \Longrightarrow\left\{\begin{array}{l}
z_{1,1}=\frac{b_{1}}{a_{1}}\left(\mathrm{e}^{a_{1}\left(t-t_{0}\right)}-1\right), \\
z_{1,2}=\frac{b_{2}}{a_{2}}\left(\mathrm{e}^{a_{2}\left(t-t_{0}\right)}-1\right),
\end{array}\right. \\
& b_{2}<0 \Longrightarrow\left\{\begin{array}{l}
z_{1,1}=\frac{b_{1}}{a_{2}}\left(\mathrm{e}^{a_{2}\left(t-t_{0}\right)}-1\right), \\
z_{1,2}=\frac{b_{2}}{a_{1}}\left(\mathrm{e}^{a_{1}\left(t-t_{0}\right)}-1\right),
\end{array}\right. \\
& z_{0}>0 \Longrightarrow\left\{\begin{array}{l}
z_{2,1}=z_{0} \mathrm{e}^{a_{1}\left(t-t_{0}\right)}, \\
z_{2,2}=z_{0} \mathrm{e}^{a_{2}\left(t-t_{0}\right)},
\end{array}\right. \\
& z_{0}<0 \Longrightarrow\left\{\begin{array}{l}
z_{2,1}=z_{0} \mathrm{e}^{a_{2}\left(t-t_{0}\right)}, \\
z_{2,2}=z_{0} \mathrm{e}^{a_{1}\left(t-t_{0}\right)} .
\end{array}\right.
\end{aligned}\right.
$$


As it happened in the foregoing Case III.5, for the determination of the domains of $Z_{1}$ and $Z_{2}$, we will use the notations $a_{0^{-}}$and $a_{0^{+}}$introduced in (121). After non difficult but elaborated computations, one gets

if $a_{1} a_{2}<0, \quad\left\{\begin{array}{l}b_{1}>0 \quad \text { or } \quad b_{1} b_{2}<0 \Longrightarrow z_{1} \in I_{1,1} \cup I_{1,2}, \\ b_{2}<0 \Longrightarrow z_{1} \in I_{1,3} \cup I_{1,4}, \\ z_{0}>0 \Longrightarrow z_{2} \in I_{2,1}^{+} \cup I_{2,2}^{+} \\ z_{0}<0 \Longrightarrow z_{2} \in I_{2,1}^{-} \cup I_{2,2}^{-}\end{array}\right.$

where

$$
\begin{gathered}
I_{1,1}=\left[\frac{b_{1}}{a_{1}}\left(\mathrm{e}^{a_{1}\left(t-t_{0}\right)}-1\right), \frac{b_{2}}{a_{0^{-}}}\left(\mathrm{e}^{a_{0^{-}}\left(t-t_{0}\right)}-1\right)\right], \\
I_{1,2}=\left[\frac{b_{1}}{a_{0^{+}}}\left(\mathrm{e}^{a_{0^{+}}\left(t-t_{0}\right)}-1\right), \frac{b_{2}}{a_{2}}\left(\mathrm{e}^{a_{2}\left(t-t_{0}\right)}-1\right)\right], \\
I_{1,3}=\left[\frac{b_{1}}{a_{0^{-}}}\left(\mathrm{e}^{a_{0^{-}}\left(t-t_{0}\right)}-1\right), \frac{b_{2}}{a_{1}}\left(\mathrm{e}^{a_{1}\left(t-t_{0}\right)}-1\right)\right], \\
I_{1,4}=\left[\frac{b_{1}}{a_{2}}\left(\mathrm{e}^{a_{2}\left(t-t_{0}\right)}-1\right), \frac{b_{2}}{a_{0^{+}}}\left(\mathrm{e}^{a_{0^{+}}\left(t-t_{0}\right)}-1\right)\right], \\
I_{2,1}^{+}=\left[z_{0} \mathrm{e}^{a_{1}\left(t-t_{0}\right)}, z_{0} \mathrm{e}^{a_{0^{-}}\left(t-t_{0}\right)}\right], \\
I_{2,2}^{+}=\left[z_{0} \mathrm{e}^{a_{0^{+}}\left(t-t_{0}\right)}, z_{0} \mathrm{e}^{a_{2}\left(t-t_{0}\right)}\right], \\
I_{2,1}^{-}=\left[z_{0} \mathrm{e}^{a_{0^{-}}\left(t-t_{0}\right)}, z_{0} \mathrm{e}^{a_{1}\left(t-t_{0}\right)}\right], \\
I_{2,2}^{-}=\left[z_{0} \mathrm{e}^{a_{2}\left(t-t_{0}\right)}, z_{0} \mathrm{e}^{a_{0^{+}}\left(t-t_{0}\right)}\right] .
\end{gathered}
$$

Finally, we apply Proposition 7 with the following identifications: $X_{1}=Z_{1}, X_{2}=Z_{2}$, and $Y_{1}=Z$. As the variation of $Z_{2}$ given by (136), (138) and (139) is easily controlled in terms of the data than of $Z_{1}$, in order to facilitate in practice the determination of the limits of integration of the integral which define the 1-PDF $f_{1}(z, t)$, we will use formula (22) rather than (21). This yields

$$
\begin{aligned}
& f_{1}(z, t) \\
& =\int_{D_{Z_{2}}} f_{B, A}\left(\frac{z_{0}\left(z-z_{2}\right)}{t-t_{0}} \frac{\ln \left|z_{2}\right|-\ln \left|z_{0}\right|}{z_{2}-z_{0}},\right. \\
& \left.\frac{\ln \left|z_{2}\right|-\ln \left|z_{0}\right|}{t-t_{0}}\right) \\
& \times \frac{1}{\left(t-t_{0}\right)^{2}} \frac{z_{0}}{z_{2}}\left|\frac{\ln \left|z_{2}\right|-\ln \left|z_{0}\right|}{z_{2}-z_{0}}\right| \mathrm{d} z_{2}, \\
& z \in D_{Z}, t>t_{0},
\end{aligned}
$$

where $D_{Z_{2}}$ is defined by (136) and (138) or (139) depending on the signs of $z_{0}$ and the product $a_{1} a_{2}$. As in Case III.5, we do not explicit the range of variation of $z$, denoted by $D_{Z}$, since its writing is cumbersome but not difficult from previous exposition.
Now, we deal with the case where $z_{0}=0$ and we keep the assumption $t>t_{0}$. For convenience, we rewrite (81) in the following equivalent form:

$$
Z(t)=Z_{1}(t) Z_{2}(t), \quad \text { where }\left\{\begin{array}{l}
Z_{1}(t)=\frac{B}{A}, \\
Z_{2}(t)=\mathrm{e}^{A\left(t-t_{0}\right)}-1 .
\end{array}\right.
$$

To apply RVT method, we fix $t: t>t_{0}$ and denote $Z_{1}=Z_{1}(t)$, $Z_{2}=Z_{2}(t)$, and $Z=Z_{1} Z_{2}=Z(t)$. To compute the PDF of $Z$, first we will determine the joint PDF of $Z_{1}$ and $Z_{2}$ by applying Theorem 5 to

$$
\begin{gathered}
X_{1}=B, \quad X_{2}=A, \quad r_{1}(b, a)=\frac{b}{a}, \\
r_{2}(a)=\mathrm{e}^{a\left(t-t_{0}\right)}-1, \quad Y_{1}=Z_{1}, \quad Y_{2}=Z_{2}, \\
s_{1}\left(z_{1}, z_{2}\right)=\frac{z_{1} \ln \left(1+z_{2}\right)}{t-t_{0}}, \quad s_{2}\left(z_{2}\right)=\frac{\ln \left(1+z_{2}\right)}{t-t_{0}} .
\end{gathered}
$$

As $\partial a / \partial z_{1}=0$, in order to compute the Jacobian $J_{2}$, it is enough to calculate the two following partial derivatives:

$$
\frac{\partial b}{\partial z_{1}}=\frac{\ln \left(1+z_{2}\right)}{t-t_{0}} \neq 0, \quad \frac{\partial a}{\partial z_{2}}=\frac{1}{\left(t-t_{0}\right)\left(1+z_{2}\right)}>0,
$$

which are well defined since $t>t_{0}$ and $1+z_{2}=\mathrm{e}^{a\left(t-t_{0}\right)} \neq 0,1$, due to by hypothesis $\mathbb{P}[\{\omega \in \Omega: A(\omega) \neq 0\}]=1$. Then, one gets

$$
\begin{aligned}
& f_{Z_{1}, Z_{2}}\left(z_{1}, z_{2}\right)= f_{B, A}\left(\frac{z_{1} \ln \left(1+z_{2}\right)}{t-t_{0}}, \frac{\ln \left(1+z_{2}\right)}{t-t_{0}}\right) \\
& \times \frac{\left|\ln \left(1+z_{2}\right)\right|}{1+z_{2}} \frac{1}{\left(t-t_{0}\right)^{2}}, \\
& z_{1,1} \leq z_{1} \leq z_{1,2}, z_{2,1} \leq z_{2} \leq z_{2,2} .
\end{aligned}
$$

Again, the values $z_{1,1}, z_{1,2}, z_{2,1}$, and $z_{2,2}$ determining the domain of variation of $Z_{1}$ and $Z_{2}$ can be computed taking into account that the function $h_{2}(a)=\mathrm{e}^{a\left(t-t_{0}\right)}-1$ is increasing on 
the whole real line in the current case where $t-t_{0}>0$. After non difficult but elaborated computations, this yields,

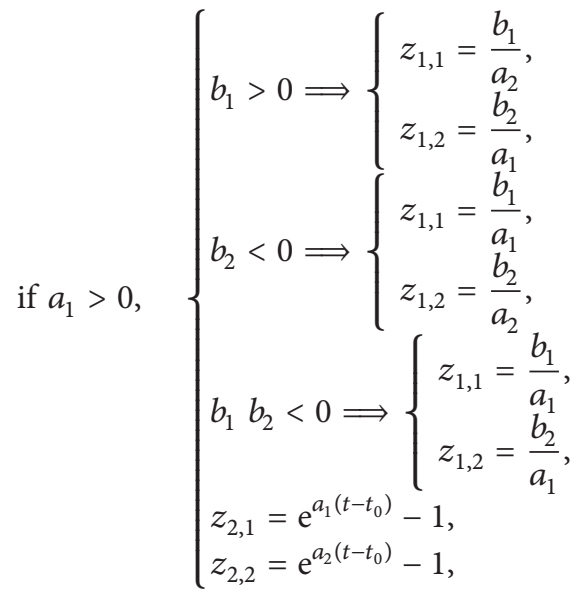

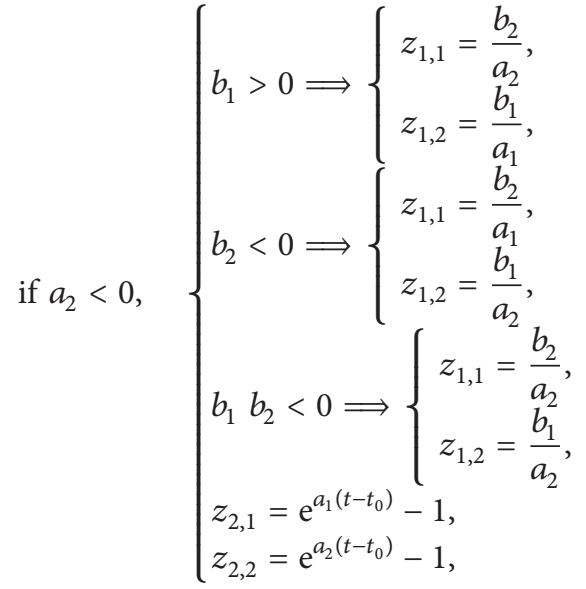

$$
\begin{aligned}
& \text { if } a_{1} a_{2}<0, \quad\left\{\begin{array}{l}
b_{1}>0 \Longrightarrow z_{1} \in I_{1,1}^{+} \cup I_{1,2}^{+}, \\
b_{2}<0 \Longrightarrow z_{1} \in I_{1,1}^{-} \cup I_{1,2}^{-}, \\
b_{1} b_{2}<0 \Longrightarrow z_{1} \in I_{1,1} \cup I_{1,2}, \\
z_{2} \in I_{2,1} \cup I_{2,2},
\end{array}\right.
\end{aligned}
$$

where

$$
\begin{gathered}
I_{1,1}^{+}=\left[\frac{b_{2}}{a_{0^{-}}}, \frac{b_{1}}{a_{1}}\right], \quad I_{1,2}^{+}=\left[\frac{b_{1}}{a_{2}}, \frac{b_{2}}{a_{0^{+}}}\right], \\
I_{1,1}^{-}=\left[\frac{b_{2}}{a_{1}}, \frac{b_{1}}{a_{0^{-}}}\right], \quad I_{1,2}^{-}=\left[\frac{b_{1}}{a_{0^{+}}}, \frac{b_{2}}{a_{2}}\right], \\
I_{1,1}=\left[\frac{b_{2}}{a_{0^{-}}}, \frac{b_{1}}{a_{0^{-}}}\right], \quad I_{1,2}=\left[\frac{b_{1}}{a_{0^{+}}}, \frac{b_{2}}{a_{0^{+}}}\right], \\
I_{2,1}=\left[\mathrm{e}^{a_{1}\left(t-t_{0}\right)}-1, \mathrm{e}^{a_{0}-\left(t-t_{0}\right)}-1\right], \\
I_{2,2}=\left[\mathrm{e}^{a_{0^{+}}\left(t-t_{0}\right)}-1, \mathrm{e}^{a_{2}\left(t-t_{0}\right)}-1\right] .
\end{gathered}
$$

Next, we apply Proposition 8 with the following identifications: $X_{1}=Z_{1}, X_{2}=Z_{2}$, and $Y_{1}=Z$. As the variation of $Z_{2}$ given by (145) and (147), which depends on the sign of $a_{1}, a_{2}$, and $a_{1} a_{2}$, is easily controlled in terms of the data than of $Z_{1}$, in order to facilitate in practice the limits of integration of the integral defining the 1-PDF $f_{1}(z, t)$, we will use formula (25) rather than (24). This yields

$$
\begin{gathered}
f_{1}(z, t)=\int_{D_{Z_{2}}} \frac{1}{\left|z_{2}\right|} f_{B, A}\left(\frac{z \ln \left(1+z_{2}\right)}{z_{2}\left(t-t_{0}\right)}, \frac{\ln \left(1+z_{2}\right)}{t-t_{0}}\right) \\
\times \frac{\left|\ln \left(1+z_{2}\right)\right|}{1+z_{2}} \frac{1}{\left(t-t_{0}\right)^{2}} \mathrm{~d} z_{2}, \\
z \in D_{Z}, t>t_{0},
\end{gathered}
$$

where $D_{Z_{2}}$ denotes the domain of variation of $Z_{2}$. Again, we do not explicit the range of variation of $Z$, denoted by $D_{Z}$, since its writing is cumbersome but simple from previous exposition.

Finally, we consider the case $t=t_{0}$ that implies $Z(t)=$ $Z\left(t_{0}\right)=z_{0}$. Therefore,

$$
f_{1}\left(z, t_{0}\right)=\delta\left(z-z_{0}\right), \quad-\infty<z<\infty .
$$

Example 23. With the aim of showing the generality of the obtained results to deal with different classes of continuous RVs, in this example, we will consider that the joint PDF of the input continuous RVs $B$ and $A$ is constructed by a copula function. Let $B$ and $A$ be two uniform continuous RV on the interval ]0,1[; that is, $B, A \sim \operatorname{Un}(] 0,1[)$. Using the FarlieGumbel-Morgenstern copula [11], we construct the random vector $(B, A)$ with joint $\mathrm{PDF}$

$$
f_{B, A}(b, a)= \begin{cases}\frac{2}{3}(2-b-a+2 b a) & \text { if } 0<b<a, 0<a<1 \\ 0 & \text { otherwise }\end{cases}
$$

hence, $B$ and $A$ are statistically dependent. As a characteristic of copula functions, notice that the two marginal distributions of $f_{B, A}(b, a)$ are just the individual distributions of $B$ and $A$. In the following, we will consider the case previously developed for $z_{0}=0$ and we will take $t_{0}=0$. First, notice that according to (141) and taking into account that $0<A, B<1$, the domains of variation of $Z_{1}$ and $Z_{2}$ are $\left.z_{1} \in\right] 0, \infty$ [ and $\left.z_{2} \in\right] 0, \mathrm{e}^{t}-1\left[\right.$, respectively; thus, $Z=Z_{1} Z_{2}$ lies on $\left.z \in\right] 0, \infty[$. However, we must refine the domain of integration $D_{Z_{2}}$ to $Z_{2}$ of (148), in such a way that the two arguments of the PDF $f_{B, A}(\cdot, \cdot)$ lie inside the interval ]0,1[, where the input continuous RVs $B$ and $A$ are defined. Thus, fixing $t>0$ and $z>0$, we must determine $z_{2}$ such as

$$
0<\frac{z \ln \left(1+z_{2}\right)}{z_{2} t}<1, \quad 0<\frac{\ln \left(1+z_{2}\right)}{t}<1
$$

hold. By (141), notice that in our context $z_{2}=\mathrm{e}^{a t}-1$, with $0<a<1$; hence, second inequality in (151) is guaranteed. As $t, z, z_{2}, \ln \left(1+z_{2}\right)>0$, the first inequality in (151) is equivalent to $\ln \left(1+z_{2}\right) / z_{2}<t / z$. Therefore, $D_{Z_{2}}$ in (148) is given by $\left.D_{Z_{2}}:\right] \min \left(\max \left(0, z_{2}^{*}\right), \mathrm{e}^{t}-1\right), \mathrm{e}^{t}-1\left[\right.$, where $z_{2}^{*}$ is the solution of the equation $\ln \left(1+z_{2}\right) / z_{2}=t / z$. At this point, notice that the function $\ln \left(1+z_{2}\right) / z_{2}$ is decreasing for $z_{2}>0$ and this 


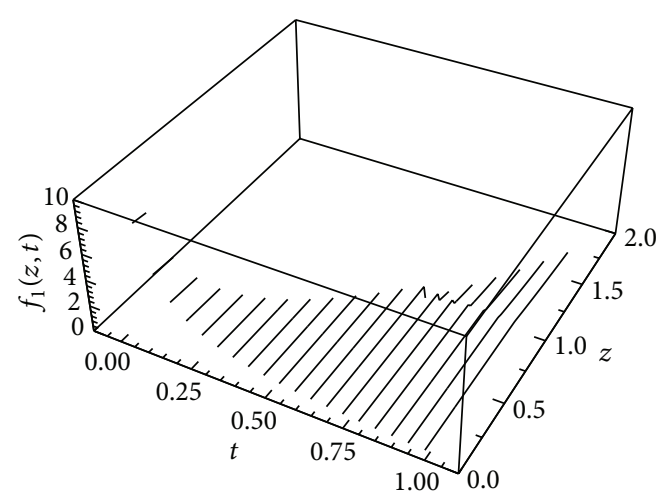

(a)

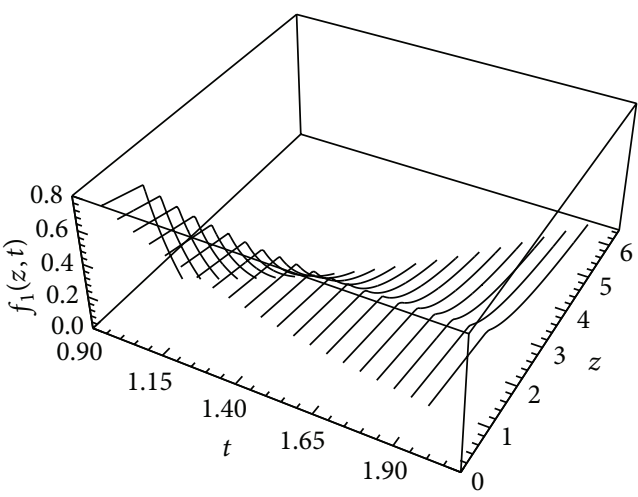

(b)

Figure 13: Plot of $f_{1}(z, t)$ given by (152) in Example 23 at different values of $t=\{0.1,0.2, \ldots, 1\}$ (a) and $t=\{1,1.1, \ldots, 2\}$ (b) in the case that $z_{0}=0$ and $t_{0}=0$ and the joint PDF of $(B, A)$ is given by (150). In order to better highlight the shape of $f_{1}(z, t)$, we have split its representation into two plots. Notice the different scales in the vertical axes for each plot.

justifies the consideration of the previous term: $\max \left(0, z_{2}^{*}\right)$. To summarize, the 1-PDF of $Z(t)$ is given by

$$
\begin{aligned}
& f_{1}(z, t)=\frac{2}{3 t^{2}} \int_{z_{2,1}}^{\mathrm{e}^{t}-1} \frac{1}{z_{2}} \frac{\ln \left(1+z_{2}\right)}{1+z_{2}} \\
& \times\left\{2-\frac{\ln \left(1+z_{2}\right)}{z_{2} t^{2}}\right. \\
&\left.\times\left(z t-z_{2} t-2 z \ln \left(1+z_{2}\right)\right)\right\} \mathrm{d} z_{2},
\end{aligned}
$$$$
t, z>0
$$

where $z_{2,1}=\min \left(\max \left(0, z_{2}^{*}\right), \mathrm{e}^{t}-1\right)$. In Figure 13, we have plotted $f_{1}(z, t)$ at different values of $t$.

5.7. Case III.7: $\left(Z_{0}, B, A\right)$ Is a Random Vector. Finally, we consider the IVP (1) and assume that every input is randomized; that is, $\left(Z_{0}, B, A\right)$ is a random vector with joint $\operatorname{PDF} f_{Z_{0}, B, A}\left(z_{0}, b, a\right)$. For convenience, we rewrite (81) in the following equivalent form:

$$
\begin{aligned}
& Z(t)=Z_{1}(t)+Z_{2}(t)+Z_{3}(t), \\
& \text { where }\left\{\begin{array}{l}
Z_{1}(t)=Z_{0} \mathrm{e}^{A\left(t-t_{0}\right)}, \\
Z_{2}(t)=\frac{B}{A} \mathrm{e}^{A\left(t-t_{0}\right)}, \\
Z_{3}(t)=-\frac{B}{A}
\end{array}\right.
\end{aligned}
$$

In order to apply RVT method, we fix $t: t>t_{0}$ and denote $Z_{1}=Z_{1}(t), Z_{2}=Z_{2}(t), Z_{3}=Z_{3}(t)$, and $Z=Z_{1}+Z_{2}+Z_{3}=$
$Z(t)$. To compute the PDF of $Z$, first we will determine the joint $\mathrm{PDF}$ of $Z_{1}, Z_{2}$, and $Z_{3}$ by applying Theorem 4 to

$$
\begin{gathered}
X_{1}=Z_{0}, \quad Y_{1}=Z_{1}, \quad r_{1}\left(z_{0}, a\right)=z_{0} \mathrm{e}^{a\left(t-t_{0}\right)}, \\
s_{1}\left(z_{1}, z_{2}, z_{3}\right)=-\frac{z_{1} z_{3}}{z_{2}}, \quad X_{2}=B, \quad Y_{2}=Z_{2} \\
r_{2}(b, a)=\frac{b}{a} \mathrm{e}^{a\left(t-t_{0}\right)}, \quad s_{2}\left(z_{2}, z_{3}\right)=-\frac{z_{3}}{t-t_{0}} \ln \left(-\frac{z_{2}}{z_{3}}\right), \\
X_{3}=A, \quad Y_{3}=Z_{3}, \quad r_{3}(b, a)=-\frac{b}{a}, \\
s_{3}\left(z_{2}, z_{3}\right)=\frac{1}{t-t_{0}} \ln \left(-\frac{z_{2}}{z_{3}}\right) .
\end{gathered}
$$

Notice that $-z_{2} / z_{3}=\mathrm{e}^{a\left(t-t_{0}\right)}>0$ so $s_{2}\left(z_{2}, z_{3}\right)$ and $s_{3}\left(z_{2}, z_{3}\right)$ are well defined as $B$ and $A$ are continuous RVs such as $\mathbb{P}[\{\omega \in \Omega: B(\omega) \neq 0\}]=1$ and $\mathbb{P}[\{\omega \in \Omega: A(\omega) \neq 0\}]=$ 1 , respectively. After some computations, it is easy to check that the absolute value of the Jacobian $J_{3}$ required to apply Theorem 4 is given by

$$
\left|J_{3}\right|=\frac{\left|z_{3}\right|}{\left(z_{2}\right)^{2}} \frac{1}{\left(t-t_{0}\right)^{2}}\left|\ln \left(-\frac{z_{2}}{z_{3}}\right)\right| \neq 0 .
$$

Notice that we have used $z_{3} \neq 0$. Therefore,

$$
\begin{gathered}
f_{Z_{1}, Z_{2}, Z_{3}}\left(z_{1}, z_{2}, z_{3}\right) \\
=f_{Z_{0}, B, A}\left(-\frac{z_{1} z_{3}}{z_{2}},-\frac{z_{3}}{t-t_{0}} \ln \left(-\frac{z_{2}}{z_{3}}\right),\right. \\
\left.\frac{1}{t-t_{0}} \ln \left(-\frac{z_{2}}{z_{3}}\right)\right) \\
\cdot \frac{\left|z_{3}\right|}{\left(z_{2}\right)^{2}} \frac{1}{\left(t-t_{0}\right)^{2}}\left|\ln \left(-\frac{z_{2}}{z_{3}}\right)\right|, \quad z_{1,1} \leq z_{1} \leq z_{1,2}, \\
z_{2,1} \leq z_{2} \leq z_{2,2}, z_{3,1} \leq z_{3} \leq z_{3,2} .
\end{gathered}
$$


Now, we will apply Proposition 9 with the following identifications: $X_{1}=Z_{1}, X_{2}=Z_{2}, X_{3}=Z_{3}$, and $Y_{1}=Z$. As the variation of $Z_{2}$ and $Z_{3}$ is easily controlled in terms of the data than of $Z_{1}$, in order to facilitate in practice the specification of the limits of integration of the integral defining the 1-PDF $f_{1}(z, t)$, we will use formula (27). This yields

$$
\begin{aligned}
& f_{1}(z, t) \\
& \begin{aligned}
=\int_{D_{Z_{3}}} \int_{D_{Z_{2}}} f_{Z_{0}, B, A}( & -\frac{\left(z-z_{2}-z_{3}\right) z_{3}}{z_{2}}, \\
& \left.-\frac{z_{3}}{t-t_{0}} \ln \left(-\frac{z_{2}}{z_{3}}\right), \frac{1}{t-t_{0}} \ln \left(-\frac{z_{2}}{z_{3}}\right)\right) \\
\times & \frac{\left|z_{3}\right|}{\left(z_{2}\right)^{2}} \frac{1}{\left(t-t_{0}\right)^{2}}\left|\ln \left(-\frac{z_{2}}{z_{3}}\right)\right| \mathrm{d} z_{2} \mathrm{~d} z_{3},
\end{aligned}
\end{aligned}
$$

for $z \in D_{Z}$ and $t>t_{0}$. As in the previous case, we do not detail the range of variation of $z, D_{Z}$, since it is very involved.

Finally, we consider the case $t=t_{0}$. From (1), one gets $Z(t)=Z\left(t_{0}\right)=Z_{0}$. Therefore,

$$
\begin{aligned}
& f_{1}\left(z_{0}, t_{0}\right) \\
& \quad=f_{Z_{0}}\left(z_{0}\right) \\
& \quad=\int_{b_{1}}^{b_{2}} \int_{a_{1}}^{a_{2}} f_{Z_{0}, B, A}\left(z_{0}, b, a\right) \mathrm{d} b \mathrm{~d} a, \quad z_{0,1} \leq z_{0} \leq z_{0,2} .
\end{aligned}
$$

Example 24. Let $\left(Z_{0}, B, A\right)$ be a three-dimensional Gaussian vector, $\eta=\left(Z_{0}, B, A\right)^{T} \sim N\left(\mu_{\eta}, \Sigma_{\eta}\right)$, where $\mu_{\eta}=\left(\mu_{1}, \mu_{2}, \mu_{3}\right)^{T}$ is a vector in $\mathbb{R}^{3}$ which represents the mean and $\Sigma_{\eta}$ is a symmetric positive definite real matrix of size $3 \times 3$ usually referred to as the variance-covariance matrix. Then, according to (157), the 1-PDF of $Z(t)$ is given by

$$
\begin{aligned}
f_{1}(z, t)= & \frac{1}{2 \pi \sqrt{2 \pi} \sqrt{\operatorname{det}\left(\Sigma_{\eta}\right)}} \\
& \times \int_{-\infty}^{\infty} \int_{-\infty}^{\infty} \mathrm{e}^{-(1 / 2)\left(\zeta-\mu_{\eta}\right)^{T}\left(\Sigma_{\eta}\right)^{-1}\left(\zeta-\mu_{\eta}\right)} \\
& \quad \times \frac{\left|z_{3}\right|}{\left(z_{2}\right)^{2}} \frac{1}{\left(t-t_{0}\right)^{2}}\left|\ln \left(-\frac{z_{2}}{z_{3}}\right)\right| \mathrm{d} z_{2} \mathrm{~d} z_{3},
\end{aligned}
$$

where

$$
\zeta=\left(\begin{array}{c}
-\frac{\left(z-z_{2}-z_{3}\right) z_{3}}{z_{2}} \\
-\frac{z_{3}}{t-t_{0}} \ln \left(-\frac{z_{2}}{z_{3}}\right) \\
\frac{1}{t-t_{0}} \ln \left(-\frac{z_{2}}{z_{3}}\right)
\end{array}\right) .
$$

In the following, we will take $t_{0}=0$ :

$$
\mu_{\eta}=\left(\begin{array}{l}
1 \\
1 \\
1
\end{array}\right), \quad \Sigma_{\eta}=\frac{1}{10}\left(\begin{array}{lll}
4 & 1 & 1 \\
1 & 4 & 1 \\
1 & 1 & 2
\end{array}\right)
$$

Now, we do not provide an explicit expression of $f_{1}(z, t)$ since it is very sophisticated. Figure 14 shows $f_{1}(z, t)$ at different values of $t(t=0,0.1,1,2)$.

\section{Some Final Remarks}

In this section, we will point out some considerations related to the practical application of RVT method in dealing with the computation of the 1-PDF.

Remark 25. Throughout this paper, we have determined the 1-PDF of the solution SP to IVP (1) in all the cases listed in Table 1. It must be pointed out that it has depended heavily on doing an appropriate choice of the involved variables when applying the RVT method. To illustrate this statement, let us consider the foregoing Case III.5 when $Z_{0}$ and $A$ are the only input continuous RVs. When we dealt with this case, we first decomposed the solution SP (81) in the form given by (116) and then we applied Theorem 5 as is shown in (117). This decomposition was carefully chosen to guarantee the successful application of Theorem 5. In fact, alternative decompositions of (81) are possible; however, they could not be adequate to achieve our goal. For example, if we rewrite (81) in the following form:

$$
\begin{aligned}
& Z(t)=Z_{1}(t)+Z_{2}(t), \\
& \text { where }\left\{\begin{array}{l}
Z_{1}(t)=Z_{0} \mathrm{e}^{A\left(t-t_{0}\right)}, \\
Z_{2}(t)=\frac{b}{A}\left(\mathrm{e}^{A\left(t-t_{0}\right)}-1\right),
\end{array}\right.
\end{aligned}
$$

then, the application of Theorem 5 to

$$
\begin{aligned}
X_{1}=Z_{0}, \quad X_{2}=A, & r_{1}\left(z_{0}, a\right)=z_{0} \mathrm{e}^{a\left(t-t_{0}\right)}, \\
r_{2}(a)=\frac{b}{a}\left(\mathrm{e}^{a\left(t-t_{0}\right)}-1\right), & Y_{1}=Z_{1}, \quad Y_{2}=Z_{2}, \\
s_{1}\left(z_{1}, z_{2}\right)= & s_{2}\left(z_{2}\right)=
\end{aligned}
$$

does not lead to fruitful results since we cannot isolate $z_{0}=s_{1}\left(z_{1}, z_{2}\right)$ and $a=s_{2}\left(z_{1}, z_{2}\right)$ and this would ruin our goal. In other cases, we can find out that two or more choices do not have the previous drawback and then the best selection will be the one which facilitates the involved computations (the easiest expression for the Jacobian, the simplest determination of the domains, etc.).

Remark 26. Although in this paper we have concentrated on the determination of the 1-PDF $f_{1}(z, t)$, which describes 


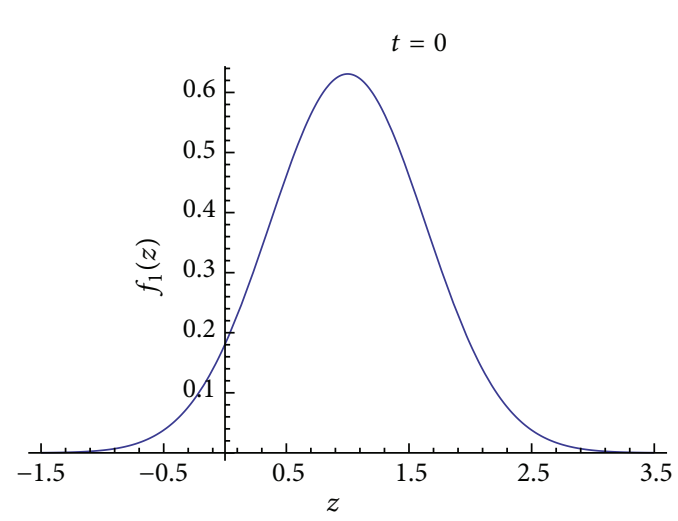

(a)

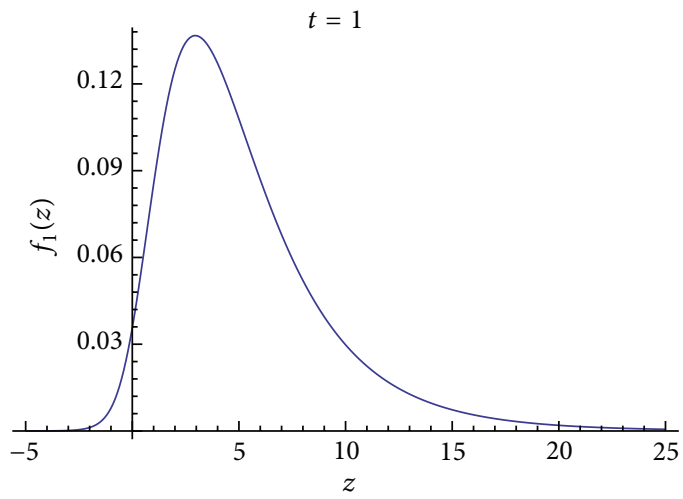

(c)

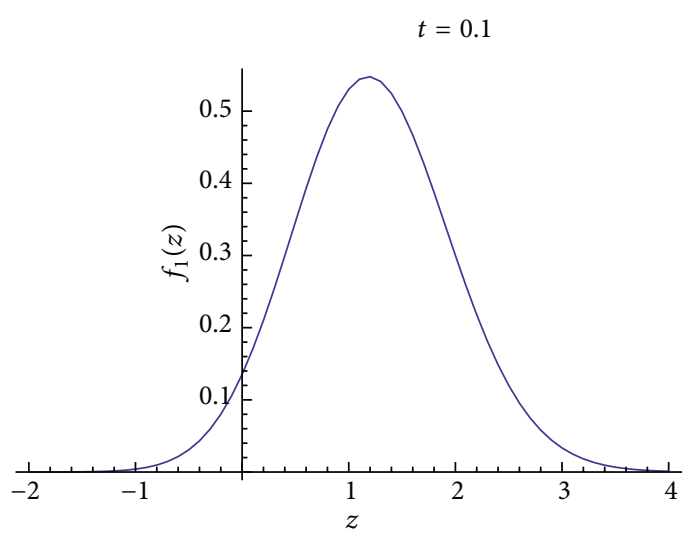

(b)

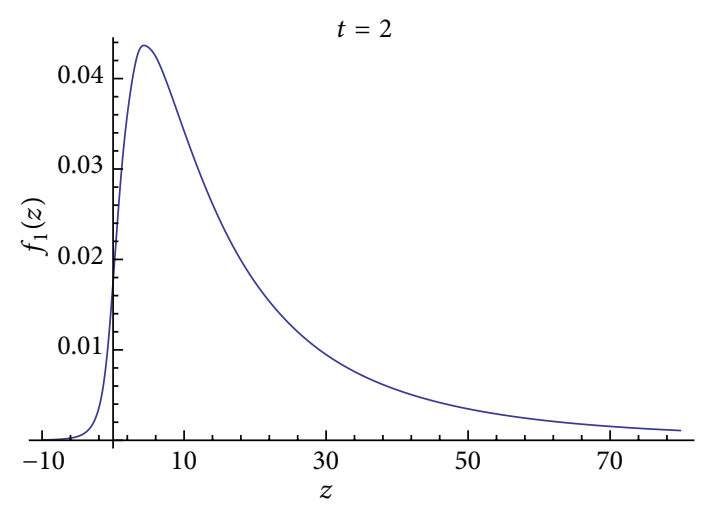

(d)

FIGURE 14: Plots of $f_{1}(z, t)$ given by (159) in Example 24 at different values of $t$ with $t_{0}=0$ in the case that $\left(Z_{0}, B, A\right)$ is a three-dimensional Gaussian vector with mean and variance-covariance matrix given by (161).

the probabilistic behaviour of the solution SP $Z(t)$ at each time instant $t$, it must be pointed out that the computation of higher PDFs is also possible and desirable. In fact, for instance, the 2-PDF, say, $f_{2}\left(z_{1}, t_{1} ; z_{2}, t_{2}\right)$ provides a whole probabilistic information of $X(t)$ in two time instants $t_{1}$ and $t_{2}$. Therefore, from it, we can calculate relevant probabilistic properties such as the correlation function

$$
\Gamma_{Z}\left(t_{1}, t_{2}\right)=\int_{-\infty}^{\infty} \int_{-\infty}^{\infty} z_{1} z_{2} f_{2}\left(z_{1}, t_{1} ; z_{2}, t_{2}\right) \mathrm{d} z_{1} \mathrm{~d} z_{2}
$$

As it can be guested, from the previous development, the computation of $f_{2}\left(z_{1}, t_{1} ; z_{2}, t_{2}\right)$ follows in broad outline that $f_{1}(z, t)$. In Example 27, we compute the 2-PDF within the framework of foregoing Case II.3 including the correlation function.

Example 27. Let us fix $t_{1}, t_{2}$ such as $t_{2}>t_{1} \geq t_{0}$ and denote $Z_{1}=Z\left(t_{1}\right)$ and $Z_{2}=Z\left(t_{2}\right)$. We want to determine the joint PDF of continuous RVs $Z_{1}$ and $Z_{2}$. For it, we will apply Theorem 5 to

$$
\begin{aligned}
& X_{1}=Z_{0}, \quad X_{2}=B, \quad r_{1}\left(z_{0}, b\right)=z_{0}+b\left(t_{1}-t_{0}\right), \\
& r_{2}\left(z_{0}, b\right)=z_{0}+b\left(t_{2}-t_{0}\right), \quad Y_{1}=Z_{1}, \quad Y_{2}=Z_{2}, \\
& s_{1}\left(z_{1}, z_{2}\right)=\frac{z_{1}\left(t_{2}-t_{0}\right)-z_{2}\left(t_{1}-t_{0}\right)}{t_{2}-t_{1}}, \\
& s_{2}\left(z_{1}, z_{2}\right)=\frac{z_{2}-z_{1}}{t_{2}-t_{1}},
\end{aligned}
$$

where the expression of the solution SP given by (56) has been considered. Now, taking into account that

$$
\begin{array}{ll}
\frac{\mathrm{d} s_{1}\left(z_{1}, z_{2}\right)}{\mathrm{d} z_{1}}=\frac{t_{2}-t_{0}}{t_{2}-t_{1}}, & \frac{\mathrm{d} s_{1}\left(z_{1}, z_{2}\right)}{\mathrm{d} z_{2}}=\frac{t_{1}-t_{0}}{t_{2}-t_{1}}, \\
\frac{\mathrm{d} s_{2}\left(z_{1}, z_{2}\right)}{\mathrm{d} z_{1}}=-\frac{1}{t_{2}-t_{1}}, & \frac{\mathrm{d} s_{2}\left(z_{1}, z_{2}\right)}{\mathrm{d} z_{2}}=\frac{1}{t_{2}-t_{1}},
\end{array}
$$

then, the involved Jacobian is given by

$$
\left|J_{2}\right|=\frac{1}{t_{2}-t_{1}}>0 .
$$


This leads to

$$
\begin{aligned}
f_{2}\left(z_{1}, t_{1} ; z_{2}, t_{2}\right) \\
=f_{Z_{1}, Z_{2}}\left(z_{1}, z_{2}\right) \\
=f_{Z_{0}, B}\left(\frac{z_{1}\left(t_{2}-t_{0}\right)-z_{2}\left(t_{1}-t_{0}\right)}{t_{2}-t_{1}}, \frac{z_{2}-z_{1}}{t_{2}-t_{1}}\right) \\
\quad \times \frac{1}{t_{2}-t_{1}}, \quad z_{1,1} \leq z_{1} \leq z_{1,2}, \quad z_{2,1} \leq z_{2} \leq z_{2,2},
\end{aligned}
$$

where

$$
\begin{array}{ll}
z_{1,1}=z_{0,1}+b_{1}\left(t_{1}-t_{0}\right), & z_{1,2}=z_{0,2}+b_{2}\left(t_{1}-t_{0}\right), \\
z_{2,1}=z_{0,1}+b_{1}\left(t_{2}-t_{0}\right), & z_{2,2}=z_{0,2}+b_{2}\left(t_{2}-t_{0}\right) .
\end{array}
$$

In spite of having computed the 2-PDF in the previous example, it must be noticed that, in general, its determination to problem (1) becomes very difficult even prohibitive, particularly the specification of the associated domains.

Remark 28. In some situations, the determination of the 1-PDF provides the full probabilistic information of the solution SP; hence, the computation of higher PDFs is not necessary. This is illustrated in the following example.

Example 29. Let the IVP be considered in Case III.1 where the only random input is the initial condition $Z_{0}$. We know that

$$
Z(t)=\left(Z_{0}+\frac{b}{a}\right) \mathrm{e}^{a\left(t-t_{0}\right)}-\frac{b}{a}
$$

Its $1-\mathrm{PDF}$ is given by (84). From it, we can compute any probabilistic information for every time instant, say $t_{1}$. Let us consider another time instant, say $t_{2}$. Notice that, in this case, the solution SP at $t_{2}$ can be represented as follows:

$$
\begin{aligned}
Z\left(t_{2}\right) & =\left(Z_{0}+\frac{b}{a}\right) \mathrm{e}^{a\left(t_{2}-t_{0}\right)}-\frac{b}{a} \\
& =\mathrm{e}^{a\left(t_{2}-t_{1}\right)}\left(Z_{0}+\frac{b}{a}\right) \mathrm{e}^{a\left(t_{1}-t_{0}\right)}-\frac{b}{a} \\
& =\mathrm{e}^{a\left(t_{2}-t_{1}\right)}\left(Z\left(t_{1}\right)+\frac{b}{a}\right)-\frac{b}{a} \\
& =\mathrm{e}^{a\left(t_{2}-t_{1}\right)} Z\left(t_{1}\right)+\frac{b}{a}\left(\mathrm{e}^{a\left(t_{2}-t_{1}\right)}-1\right) .
\end{aligned}
$$

From this expression, we see that the behaviour of the solution $Z(t)$ at the time instant $t_{2}$ is deterministically given by (a linear transformation of) $Z\left(t_{1}\right)$. Therefore, the computation of the 2-PDF is not required. This can be confirmed from another point of view. Let us assume without loss of generality that the expectation of the initial condition is zero: $E\left[Z_{0}\right]=0$ and its variance is $\sigma_{Z_{0}}^{2}>0$. From (170), it is easy to check that

$$
\begin{aligned}
& E\left[Z\left(t_{i}\right)\right]=\frac{b}{a} \mathrm{e}^{a\left(t_{i}-t_{0}\right)}-\frac{b}{a}, \quad i=1,2, \\
& \sigma_{Z\left(t_{i}\right)}^{2}=\sigma_{Z_{0}}^{2} \mathrm{e}^{2 a\left(t_{i}-t_{0}\right)}, \quad i=1,2, \\
& E\left[Z\left(t_{1}\right) Z\left(t_{2}\right)\right]=\sigma_{Z_{0}}^{2} \mathrm{e}^{a\left(t_{2}+t_{1}-2 t_{0}\right)} \\
& +\left(\frac{b}{a}\right)^{2}\left(\mathrm{e}^{a\left(t_{2}+t_{1}-2 t_{0}\right)}-\mathrm{e}^{a\left(t_{2}-t_{0}\right)}\right. \\
& \left.-\mathrm{e}^{a\left(t_{1}-t_{0}\right)}+1\right) .
\end{aligned}
$$

Then, the correlation coefficient function is given by

$$
\rho_{Z\left(t_{1}\right), Z\left(t_{2}\right)}=\frac{E\left[Z\left(t_{1}\right) Z\left(t_{2}\right)\right]-E\left[Z\left(t_{1}\right)\right] E\left[Z\left(t_{2}\right)\right]}{\sigma_{Z\left(t_{1}\right)} \sigma_{Z\left(t_{2}\right)}}=1 .
$$

\section{Conclusions}

In this paper, we have determined the first probability density function (1-PDF) of the solution stochastic process of the linear random differential equation taking advantage of random variable transformation (RVT) method. The study has been made in a systematic way in order to facilitate and clarify the development and exposition of the results as well as to facilitate their practical use. The wide range of the exhibited examples, that include both standard and non-standard probabilistic distributions, show the ability of RVT technique to deal with the computation of the 1-PDF to models based on linear random differential equations. Notice that throughout the paper no independence among the random parameters has been assumed in order to achieve general results.

\section{Conflict of Interests}

The authors declare that there is no conflict of interests regarding the publication of this paper.

\section{Acknowledgments}

This work has been partially supported by the Ministerio de Economía y Competitividad under Grant no. DPI2010-20891C02-01 and Universitat Politècnica de València under Grant no. PAID06-11-2070.

\section{References}

[1] T. T. Soong, Random Differential Equations in Science and Engineering, vol. 103, Academic Press, New York, NY, USA, 1973, Mathematics in Science and Engineering.

[2] A. Hussein and M. M. Selim, "Solution of the stochastic radiative transfer equation with Rayleigh scattering using RVT technique," Applied Mathematics and Computation, vol. 218, no. 13, pp. 7193-7203, 2012. 
[3] A. Hussein and M. M. Selim, "Solution of the stochastic transport equation of neutral particles with anisotropic scattering using RVT technique," Applied Mathematics and Computation, vol. 213, no. 1, pp. 250-261, 2009.

[4] M. El-Tawil, W. El-Tahan, and A. Hussein, "A proposed technique of SFEM on solving ordinary random differential equation," Applied Mathematics and Computation, vol. 161, no. 1, pp. 35-47, 2005.

[5] M. El-Tawil, W. El-Tahan, and A. Hussein, "Using FEM-RVT technique for solving a randomly excited boundary differential equation with a random operator," Applied Mathematics and Computation, vol. 187, no. 2, pp. 856-867, 2007.

[6] M. A. El-Tawil, "The approximate solutions of some stochastic differential equations using transformations," Applied Mathematics and Computation, vol. 164, no. 1, pp. 167-178, 2005.

[7] J. S. Simonoff, Smoothing Methods in Statistics, Springer Series in Statistics, Springer, New York, NY, USA, 1996.

[8] A. Papoulis, Probability, Random Variables, and Stochastic Processes, McGraw-Hill, New York, NY, USA, 1965.

[9] G. Casella and R. Berger, Statistical Inference, Books/Cole, New York, NY, USA, 2002.

[10] M. Abramowitz and I. A. Stegun, Eds., Handbook of Mathematical Functions, with Formulas, Graphs, and Mathematical Tables, Dover, New York, NY, USA, 1972.

[11] R. B. Nelsen, An Introduction to Copulas, vol. 139 of Lecture Notes in Statistics, Springer, New York, NY, USA, 1999. 


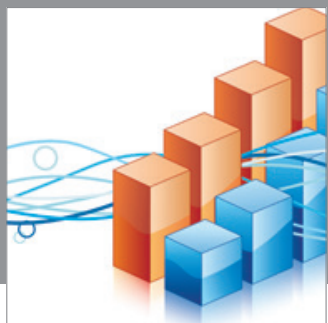

Advances in

Operations Research

mansans

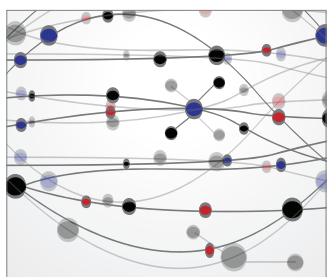

The Scientific World Journal
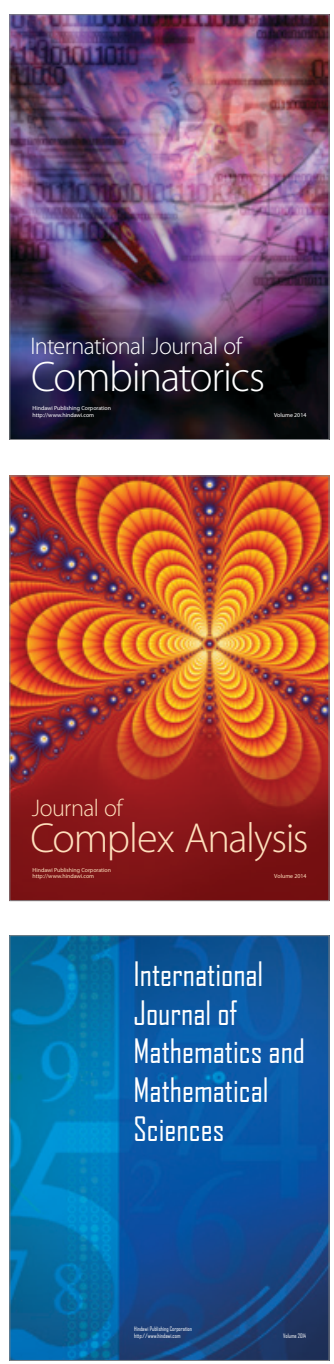
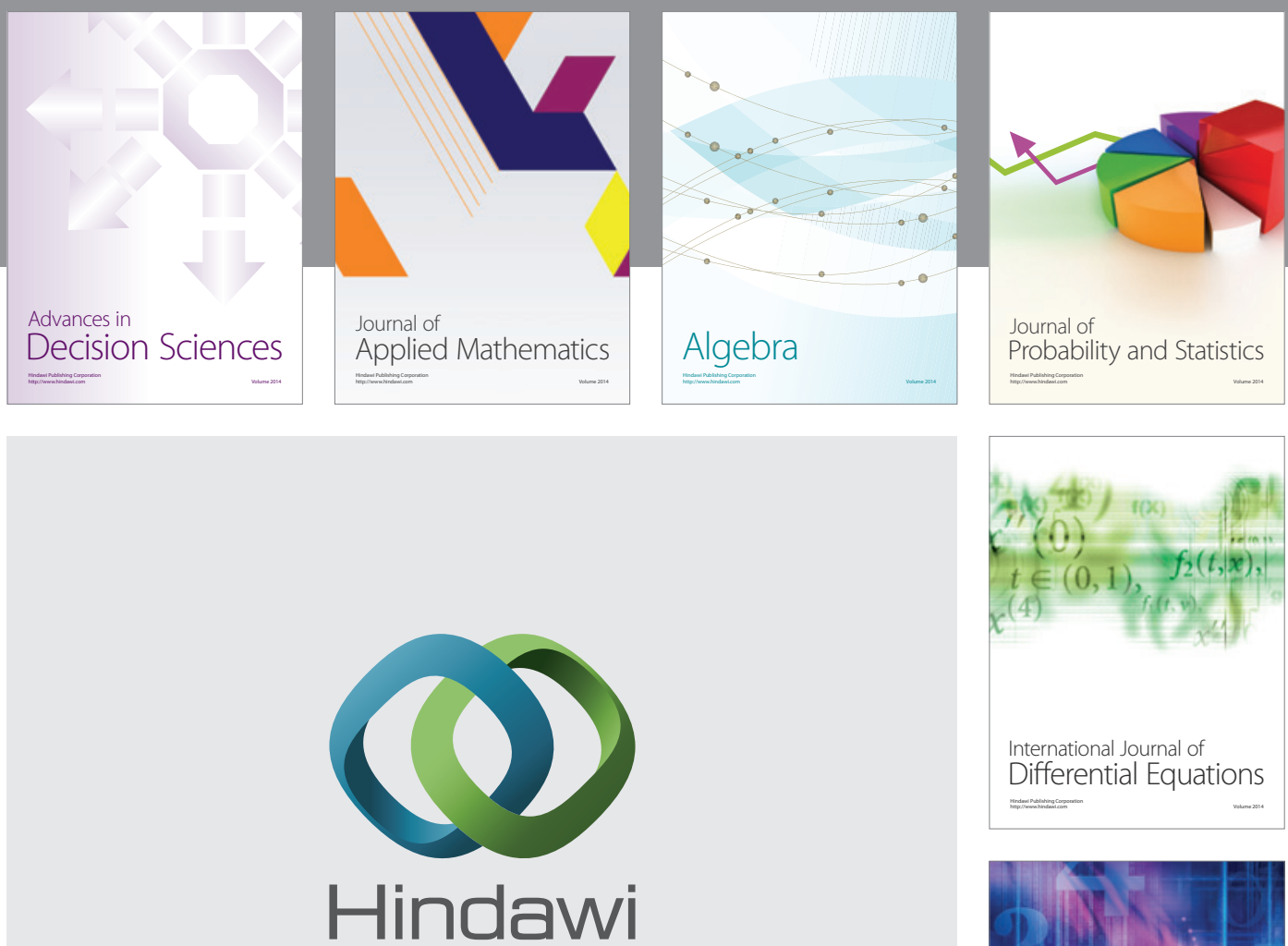

Submit your manuscripts at http://www.hindawi.com
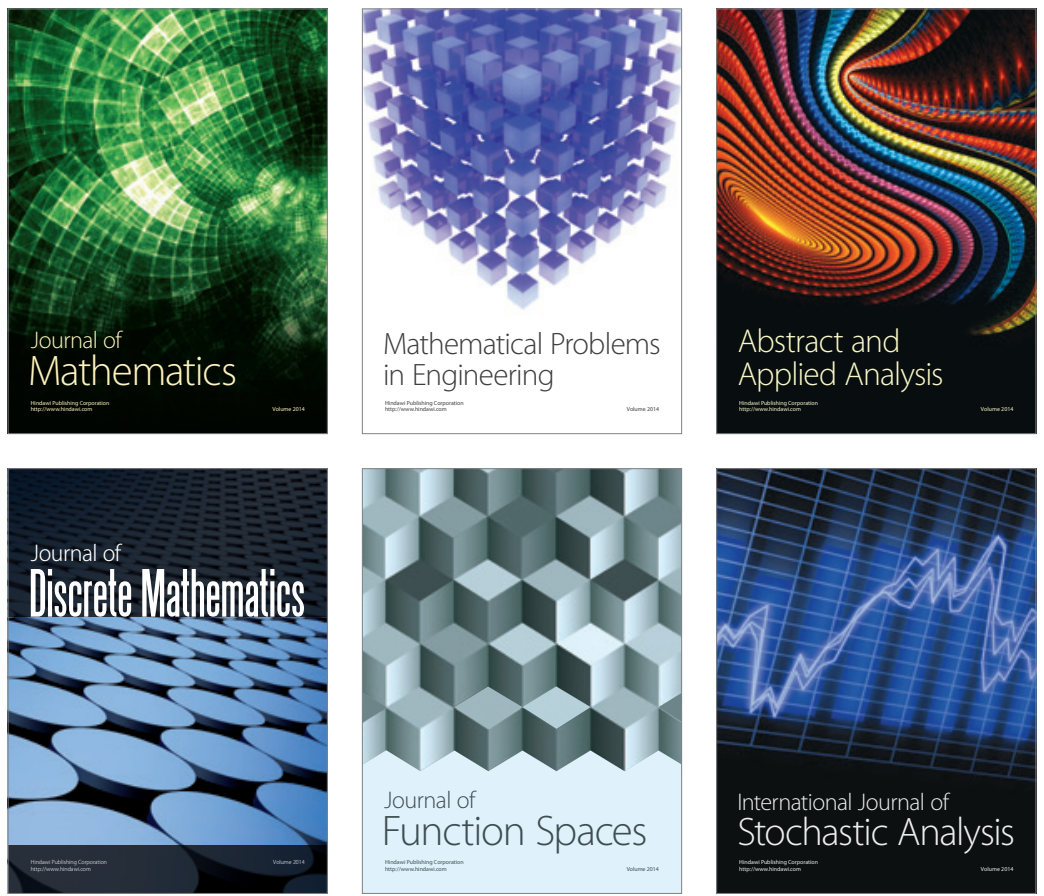

Journal of

Function Spaces

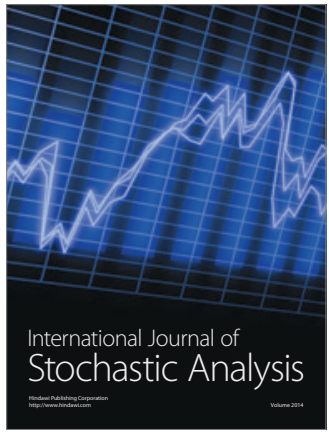

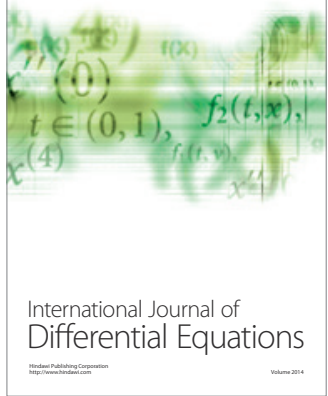
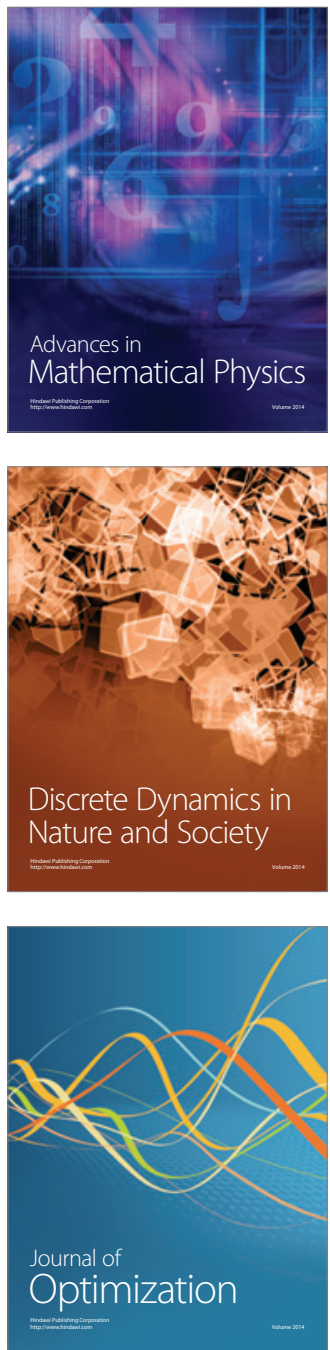